\title{
A UNITARY INVARIANT IN RIEMANNIAN GEOMETRY
}

\author{
ALAIN CONNES
}

Dédié à Michel Dubois-Violette

On nous a tant assubjectis aux cordes

que nous n'avons plus

de franches allures.

Montaigne - Livre I, Chapitre 26

\begin{abstract}
We introduce an invariant of Riemannian geometry which measures the relative position of two von Neumann algebras in Hilbert space, and which, when combined with the spectrum of the Dirac operator, gives a complete invariant of Riemannian geometry. We show that the new invariant plays the same role with respect to the spectral invariant as the Cabibbo-KobayashiMaskawa mixing matrix in the Standard Model plays with respect to the list of masses of the quarks.
\end{abstract}

\section{CONTENTS}

1. Introduction

2. The CKM matrix

2.1. Pair of maximal abelian subalgebras with labeled idempotents

2.2. Generic representations of the free product $M \star N$

2.3. The relative spectrum $\operatorname{Spec}_{N}(M)$

2.4. The CKM matrix and Fourier transform

3. Relative position of the continuum and the discrete

3.1. The Frechet manifolds $\mathbf{H}_{m}(\Lambda), \mathbf{P}_{m}(\Lambda)$ and $\mathbf{G r}_{m}(\Lambda)$

3.2. Injectivity of the map to hermitian forms

3.3. The relative spectrum $\operatorname{Spec}_{N}(M)$

3.4. Multiplicity for the discrete algebra

4. The unitary (ckm) invariant of Riemannian manifolds

4.1. Completeness of the invariant

4.2. The spectral meaning of points

4.3. The characterization of the range

5. The Sunada examples

References 


\section{INTRODUCTION}

It is well known since the original result of Milnor [16], that the spectral invariants i.e. the spectrum of operators like the Laplacian or the Dirac operator, are not complete invariants of Riemannian geometry. The goal of this paper is to describe an additional invariant which measures the relative position of two von Neumann algebras in Hilbert space, and which, when combined with the spectrum of the Dirac operator, gives a complete invariant of Riemannian geometry. We shall moreover show that the new invariant plays the same role with respect to the spectral invariant as the Cabibbo-Kobayashi-Maskawa (CKM) matrix in the Standard Model [5] plays with respect to the list of masses of the quarks.

We shall first recall the role of the CKM matrix and explain, in $₫ 2$ its conceptual meaning, whose mathematical side can in fact be traced back to the nineteenth century [24, 25], 1], 3], 12. As we shall see, it encodes the relative position of two maximal abelian von Neumann algebras $M$ and $N$ acting in three dimensional Hilbert space, and the construction easily extends to the case of arbitrary finite dimension $n$. At first the invariant is a unitary matrix $C_{x y}$ with line index $x \in$ $\operatorname{Spec}(M)$ and column index $y \in \operatorname{Spec}(N)$. We then show how to eliminate, by considering the set of lines of the matrix, the labeling by the spectrum of $M$ and obtain an invariant $\operatorname{Spec}_{N}(M)$ of the relative position of $M$ with respect to $N$ which is unaltered under automorphisms of $M$.

While our motivation comes from the example of the CKM matrix in the Standard Model another important source of examples comes from Popa's theory of commuting squares [19] which plays a central role in the construction of subfactors. The special case of commuting squares means that the absolute values $\left|C_{x y}\right|$ of all matrix elements $C_{x y}$ are equal (to $1 / \sqrt{N}$ ) and this special case gives rise to many interesting examples and questions (cf. 14, [17).

In $₫ 3$ we extend this CKM-invariant to the infinite dimensional case and define a complete invariant of the relative position of two commutative von Neumann algebras, a discrete one and a continuous one, acting in the same (separable) Hilbert space. By a result of von Neumann ( $c f$. Theorem 3.1 below) there is, up to isomorphism, only one commutative von Neumann algebra $M$ which is "continuous" i.e. has no minimal projection. Moreover, for a given multiplicity $m \in \mathbb{N}$ there is a unique (up to unitary equivalence) representation of $M$ as operators in Hilbert space with multiplicity $m$. The position of $M$ relative to a discrete von Neumann algebra $N$ (corresponding to diagonal operators for an orthonormal basis) is measured by the invariant $\operatorname{Spec}_{N}(M)$ which is a direct generalization of the CKM matrix. It is given by a measured section of a specific line bundle on a projective space of positive hermitian forms $\rho_{\lambda \kappa}$ of rank $m$ where $m$ is the multiplicity of the continuous von Neumann algebra $M$. The labels $\lambda, \kappa$ of the components of the hermitian form are elements $\lambda, \kappa \in \operatorname{Spec}(D)$ in the spectrum of the discrete von Neumann algebra $N$. In the case of interest, the discrete von Neumann algebra is generated by an unbounded self-adjoint operator $D$ and the labels can be viewed 1$]$ as real numbers $\lambda, \kappa \in \operatorname{Spec}(D) \subset \mathbb{R}$.

The whole information on a Riemannian geometry can be encoded in a pair consisting of

\footnotetext{
${ }^{1}$ modulo the issue of the multiplicity of eigenvalues.
} 
- The spectrum of the Dirac operator

- The relative position of two commutative von Neumann algebras, a discrete one and a continuous one.

The completeness of the invariant is closely related to (and follows easily from) the embedding technique of [2]. The difficult part gives a characterization of the spectral triples thus obtained. We shall then recall in $\$ 4$ the main result of 10 which gives a characterization of the spectral triples corresponding to Riemannian geometries. We end with the computation (\$5) of the invariant in a few examples, but this part is still wanting for more convincing applications.

\section{THE CKM MATRIX}

In the Standard Model giving the masses of the quarks does not suffice to specify all parameters involved in their Yukawa couplings. The missing parameters are the content of the CKM-mixing matrix which we now describe. The weak isospin group $\mathrm{SU}(2)$, which is the gauge group of the weak interactions, relates together the mass eigenstates of the up quarks with those of the down quarks and the corresponding basis (only given up to phase) have a mismatch which is expressed by a mixing matrix $C_{\lambda \kappa}$ whose indices $\lambda, \kappa$ label the three generations of quarks. This matrix appears in the terms of the lagrangian such as:

$$
\frac{i g}{2 \sqrt{2}} W_{\mu}^{+}\left(\bar{u}_{j}^{\lambda} \gamma^{\mu}\left(1+\gamma^{5}\right) C_{\lambda \kappa} d_{j}^{\kappa}\right)+\frac{i g}{2 \sqrt{2}} W_{\mu}^{-}\left(\bar{d}_{j}^{\kappa} C_{\kappa \lambda}^{\dagger} \gamma^{\mu}\left(1+\gamma^{5}\right) u_{j}^{\lambda}\right)
$$

It is responsible for the flavour-changing weak decays. The knowledge of the CKMmatrix complements the list of quark masses to specify the Yukawa couplings in the Standard Model. The terminology CKM-matrix refers to the names of N. Cabibbo who first treated the case of two generations and of M. Kobayashi and T. Maskawa who treated the case of three generations.

To be more specific for 2 generations, the matrix $C$ depends on just the Cabibbo angle $\theta_{c}$, and is given by

$$
C=\left[\begin{array}{cc}
\cos \theta_{c} & \sin \theta_{c} \\
-\sin \theta_{c} & \cos \theta_{c}
\end{array}\right] .
$$

For 3 generations the matrix $C$ has the more complicated form

$$
C=\left[\begin{array}{lll}
C_{u d} & C_{u s} & C_{u b} \\
C_{c d} & C_{c s} & C_{c b} \\
C_{t d} & C_{t s} & C_{t b}
\end{array}\right]
$$

The matrix $C$ depends upon the 3 angles $\theta_{1}, \theta_{2}, \theta_{3}$ and a complex phase $\delta$. Let $c_{i}=\cos \theta_{i}, s_{i}=\sin \theta_{i}$, and $e_{\delta}=\exp (i \delta)$, then

$$
C=\left[\begin{array}{ccc}
c_{1} & -s_{1} c_{3} & -s_{1} s_{3} \\
s_{1} c_{2} & c_{1} c_{2} c_{3}-s_{2} s_{3} e_{\delta} & c_{1} c_{2} s_{3}+s_{2} c_{3} e_{\delta} \\
s_{1} s_{2} & c_{1} s_{2} c_{3}+c_{2} s_{3} e_{\delta} & c_{1} s_{2} s_{3}-c_{2} c_{3} e_{\delta}
\end{array}\right]
$$

The mathematical treatment of the corresponding matrix problem in arbitrary dimension goes back to the nineteenth century [24, [25], 11, [3], 12]. In this section we shall explain its conceptual meaning in terms which will then be extended to the infinite dimensional case. 


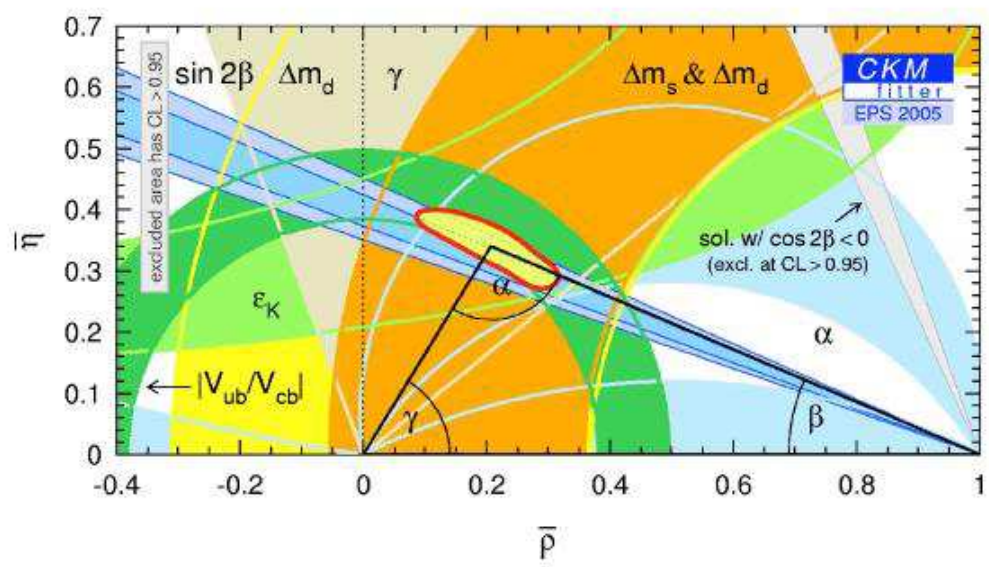

FiguRE 1. Experimental information on the CKM matrix (noted $V_{x y}$ instead of $C_{x y}$ ).

\subsection{Pair of maximal abelian subalgebras with labeled idempotents.}

By construction the CKM matrix $C_{\lambda \kappa}$ conjugates two orthonormal basis of the same three dimensional Hilbert space, but each of the basis elements are only defined up to a phase. Thus $C_{\lambda \kappa}$ is a specific representative of a double coset in the 4-dimensional coset space

$$
\Delta_{1} \backslash \mathrm{SU}(3) / \Delta_{1}=\Delta \backslash \mathrm{U}(3) / \Delta
$$

where $\Delta_{1}(\operatorname{resp} \Delta)$ is the group of unimodular diagonal matrices in $\mathrm{SU}(3)$ (resp. of diagonal matrices in $\mathrm{U}(3))$. Up to the right multiplication by the diagonal matrix

$$
\left[\begin{array}{ccc}
1 & 0 & 0 \\
0 & -1 & 0 \\
0 & 0 & -1
\end{array}\right]
$$

and assuming $\left.\theta_{j} \in\right] 0, \pi / 2$ [ the matrix $C_{\lambda \kappa}$ is the only element in the double coset of $C$ in $\Delta \backslash \mathrm{U}(3) / \Delta$ whose first line and column are having positive entries. We shall now use the same normalization condition in general.

Let $\mathcal{H}$ be a Hilbert space of finite dimension $n$ and $M, N$ be two (discrete) maximal abelian subalgebras of $\mathcal{L}(\mathcal{H})$ with labeled minimal idempotents $e_{j} \in M, f_{j} \in N$ for $j \in\{1, \ldots, n\}$.

Lemma 2.1. Assume that $e_{i} f_{j} \neq 0$ if $i$ or $j$ is 1 . Then there exists, up to an overall phase factor, a unique pair of orthonormal basis $\left(\xi_{j}\right)$ and $\left(\eta_{j}\right)$ of $\mathcal{H}$ such that

a) $e_{j} \xi_{j}=\xi_{j}$ and $f_{j} \eta_{j}=\eta_{j}$ for all $j \in\{1, \ldots, n\}$.

b) $\left\langle\xi_{j}, \eta_{i}\right\rangle>0$ if $i=1$ or $j=1$.

There exists a unique unitary operator $U \in \mathcal{L}(\mathcal{H})$ such that $U \xi_{j}=\eta_{j}$ for all $j$. One has $U e_{j} U^{*}=f_{j}$ for all $j \in\{1, \ldots, n\}$.

Proof. Let us choose a unit vector $\xi_{1}$ with $e_{1} \xi_{1}=\xi_{1}$. Then there exists, since $e_{1} f_{1} \neq 0$, a unique vector $\eta_{1}$ with $f_{1} \eta_{1}=\eta_{1}$ and such that $\left\langle\xi_{1}, \eta_{1}\right\rangle>0$. The 
conditions a) and b) then uniquely fix the vectors $\xi_{j}$ and $\eta_{j}$ for $j>1$. Since the pair of orthonormal basis $\xi_{j}$ and $\eta_{j}$ is unique up to an overall phase the operator $U$ is unique. The last assertion follows from $U \xi_{j}=\eta_{j}$.

Let us check that the number of degrees of freedom is the right one. Assume given the positive scalars

$$
\alpha_{j}=\left\langle\xi_{j}, \eta_{1}\right\rangle, \quad \beta_{i}=\left\langle\xi_{1}, \eta_{i}\right\rangle, \beta_{1}=\alpha_{1}, \quad \sum \alpha_{j}^{2}=\sum \beta_{j}^{2}=1 .
$$

This fixes the coordinates of $\eta_{1}$ in the given basis $\xi_{j}$. The only constraints on $\eta_{2}$ are that $\left\|\eta_{2}\right\|=1, \eta_{2} \perp \eta_{1},\left\langle\xi_{1}, \eta_{2}\right\rangle=\beta_{2}$. Thus the freedom of choice is a point in

$$
S_{2}=\left\{\eta \in \mathcal{H} \mid\|\eta\|=1, \eta \perp \eta_{1},\left\langle\xi_{1}, \eta\right\rangle=\beta_{2}\right\}
$$

and more generally one gets for the inductive choice of $\eta_{k}$,

$$
S_{k}=\left\{\eta \in \mathcal{H} \mid\|\eta\|=1, \eta \perp \eta_{j}, \forall j<k,\left\langle\xi_{1}, \eta\right\rangle=\beta_{k}\right\}
$$

With $\operatorname{dim} \mathcal{H}=n$ one gets that $S_{k}$ is the intersection of the unit sphere with a complex affine 2 subspace of dimension $n-k$. Thus (if non-empty) its real dimension is $2(n-k)-1$. Thus the degrees of freedom come from the $2 n-1$ scalars (4) which fulfill 2 conditions and hence give real dimension $2 n-3$ and then the sum of the $2(n-k)-1$ for $k$ between 2 and $n-1$. This gives $(n-1)^{2}$ real parameters as expected from the dimensions $n^{2}-1$ of $\mathrm{SU}(n)$ and $n-1$ of the group of diagonal unimodular unitaries.

\subsection{Generic representations of the free product $M \star N$.}

Let $M$ and $N$ be commutative von Neumann algebras of dimension $n<\infty$ and $e_{M} \in M, e_{N} \in N$ minimal idempotents, or equivalently elements of the spectrum. We let $M \star N$ be the $C^{*}$-algebra free product of $M$ and $N$. Thus giving a Hilbert space representation of $M \star N$ is the same as giving a pair of representations of $M$ and $N$ in the same Hilbert space.

Definition 2.2. We say that a representation $\pi$ of the free product $M \star N$ in a Hilbert space $\mathcal{H}$ of dimension $n$ is generic when the states $y \mapsto \operatorname{Tr}\left(\pi\left(e_{M} y\right)\right)$ on $N$ and $x \mapsto \operatorname{Tr}\left(\pi\left(x e_{N}\right)\right)$ on $M$ are faithful.

This implies that the restriction of $\pi$ to $M$ and $N$ are isomorphisms with maximal abelian subalgebras of $\mathcal{L}(\mathcal{H})$.

Proposition 2.3. The generic representations of the free product $M \star N$ in a Hilbert space $\mathcal{H}$ of dimension $n$ are classified up to unitary equivalence by matrices $C_{x y}$ with $x \in \operatorname{Spec}(M), y \in \operatorname{Spec}(N)$ such that

- $C$ is a unitary matrix

- The line and column of $C$ corresponding to $e_{M}$ and $e_{N}$ are strictly positive.

Proof. Let us first show how to associate a matrix $C(\pi)$ to a generic representation $\pi$ of the free product $M \star N$. We choose two unit vectors $\xi, \eta \in \mathcal{H}$ such that:

$$
\pi\left(e_{M}\right) \xi=\xi, \quad \pi\left(e_{N}\right) \eta=\eta, \quad\langle\xi, \eta\rangle>0 .
$$

For each $x \in \operatorname{Spec}(M)$ there is a unique corresponding minimal projection $e_{x} \in M$ and a unique unit vector $\xi_{x} \in \mathcal{H}$ such that $e_{x} \xi_{x}=\xi_{x}$ while $\left\langle\xi_{x}, \eta\right\rangle>0$. A similar

\footnotetext{
${ }^{2} \mathrm{By}$ convention the inner product is antilinear in the first variable.
} 
statement holds for $N$, with $f_{y} \eta_{y}=\eta_{y}$ while $\left\langle\xi, \eta_{y}\right\rangle>0$. We then define the matrix $C$ as follows

$$
C_{x y}=\left\langle\xi_{x}, \eta_{y}\right\rangle, \quad \forall x \in \operatorname{Spec}(M), y \in \operatorname{Spec}(N) .
$$

The choice of the pair $(\xi, \eta)$ is only unique up to an overall phase i.e. up to the modification: $(\xi, \eta) \rightarrow(\lambda \xi, \lambda \eta)$ with $|\lambda|=1$. All the vectors $\xi_{x}$ and $\eta_{y}$ get multiplied by $\lambda$ and this does not affect (8). This shows that the matrix $C$ is an invariant of the representation $\pi$. One has

$$
\sum_{x} \bar{C}_{x y_{1}} C_{x y_{2}=}=\sum_{x}\left\langle\eta_{y_{1}}, \xi_{x}\right\rangle\left\langle\xi_{x}, \eta_{y_{2}}\right\rangle=\left\langle\eta_{y_{1}}, \eta_{y_{2}}\right\rangle=\delta_{y_{1}, y_{2}}
$$

and similarly

$$
\sum_{y} C_{x_{1} y} \bar{C}_{x_{2} y}=\sum_{y}\left\langle\xi_{x_{1}}, \eta_{y}\right\rangle\left\langle\eta_{y}, \xi_{x_{2}}\right\rangle=\left\langle\xi_{x_{1}}, \xi_{x_{2}}\right\rangle=\delta_{x_{1}, x_{2}} .
$$

This shows that the matrix $C$ is unitary. The second property of lines and columns of $C$ is true by construction. Let us first show that $C(\pi)$ is a complete invariant. For this we define a "model" $\pi_{C}$ for each $C$ and show that the given representation $\pi$ is unitarily equivalent to $\pi_{C}$ for $C=C(\pi)$. This will also show that all invariants $C$ are obtained.

To construct $\pi_{C}$ we take $\ell^{2}(\operatorname{Spec}(N))$ with the diagonal action of $N$, and we define vectors $\zeta_{x}$ indexed by $x \in \operatorname{Spec}(M)$ by

$$
\left\langle\zeta_{x}, \epsilon_{y}\right\rangle=C_{x y}, \quad \forall y \in \operatorname{Spec}(N)
$$

where $\epsilon_{y}$ is the canonical basis of $\ell^{2}(\operatorname{Spec}(N))$. The vectors $\zeta_{x}$ form an orthonormal basis of $\ell^{2}(\operatorname{Spec}(N))$ and one can thus define the action of $M$ on $\ell^{2}(\operatorname{Spec}(N))$ as the diagonal action in this basis. Combining this with the canonical representation of $N$ in $\ell^{2}(\operatorname{Spec}(N))$ we thus obtain a representation $\pi_{C}$ of the free product $M \star N$ in the Hilbert space $\ell^{2}(\operatorname{Spec}(N))$. One checks using (9) that the corresponding $C\left(\pi_{C}\right)$ is $C$.

Starting from a given representation $\pi$ in $\mathcal{H}$ we use Lemma 2.1 and get the corresponding orthonormal basis $\xi_{x}$ and $\eta_{y}$ as above. We let $V$ be the unitary map from $\mathcal{H}$ to $\ell^{2}(\operatorname{Spec}(N))$ with $V \eta_{y}=\epsilon_{y}$. One then has, using (8) and (9) that $V \xi_{x}=\zeta_{x}$ which gives the unitary equivalence $\pi \sim \pi_{C(\pi)}$.

Remark 2.4. Note that the matrix $C$ is relating two different spaces $\ell^{2}(\operatorname{Spec}(M))$ and $\ell^{2}(\operatorname{Spec}(N))$. Indeed there is no a priori identification of $\operatorname{Spec}(M)$ with $\operatorname{Spec}(N)$. Note also that the square of the absolute value $\left|C_{x y}\right|^{2}$ is given by the simple expression $\left|C_{x y}\right|^{2}=\operatorname{Tr}\left(e_{x} f_{y}\right)$ in terms of the minimal projections of $M$ and $N$. In general the knowledge of the absolute values $\left|C_{x y}\right|$ does not suffice to recover $C$ and for instance the case $\left|C_{x y}\right|^{2}=1 / n$ for all $x, y$ corresponds to the mutually commuting case of [19], Definition 2.2.

\subsection{The relative spectrum $\operatorname{Spec}_{N}(M)$.}

In the construction of $\$ 2.2$, the entries of the matrix $C$ are labeled by the spectra of $M$ and $N$. We shall now show how to eliminate the labeling coming from the spectrum of $M$. The resulting invariant will give the relative position of $M$ viewed as a subalgebra of $\mathcal{L}(\mathcal{H})$ relative to $N$ viewed as a von Neumann algebra with known spectrum. In order to eliminate the parameter $x \in \operatorname{Spec}(M)$ we take the range of the function from $\operatorname{Spec}(M)$ to $N$ which assigns to $x \in \operatorname{Spec}(M)$ the corresponding 
line $C_{x y}, y \in \operatorname{Spec}(N)$ of the matrix $C$. Since $N$ is canonically the algebra of complex valued functions on $\operatorname{Spec}(N)$ one can view a line $\left(C_{x y}\right)_{y \in \operatorname{Spec}(N)}$ as an element of $N$ which we denote by $C_{x}, \bullet$ We define

$$
G(\pi)=\left\{C_{x,} \in N \mid x \in \operatorname{Spec}(M)\right\} \subset N .
$$

Since $C$ is a unitary matrix its lines belong to the unit sphere in $N$ defined by

$$
S_{\mathbb{C}}^{N}=\left\{\left.z \in N\left|\sum_{y}\right| z_{y}\right|^{2}=1\right\}
$$

Thus $\Sigma=G(\pi)$ is a subset of the unit sphere. One recovers the matrix $C$ from the subset just by taking the coordinates of its $n$ elements. This is not yet invariantly defined since we needed the base points $e_{M}$ and $e_{N}$ as well as the generic condition. In order to eliminate this choice of base points, we consider the action, by multiplication, of the unitary group $\mathcal{U}(N)$ on the projective space $\mathbb{P}_{N}=S_{\mathbb{C}}^{N} / \mathrm{U}(1)$. We let $p: S_{\mathbb{C}}^{N} \rightarrow \mathbb{P}_{N}$ be the projection.

Definition 2.5. We define the relative spectrum of $M$ relative to $N$ as

$$
\operatorname{Spec}_{N}(M)=p(G(\pi)) \subset \mathbb{P}_{N},
$$

viewed as a subset defined up to the gauge action of the unitary group $\mathcal{U}(N)$.

The gauge ambiguity shows up in the choice of an isomorphism of the representation of $N$ in $\mathcal{H}$ with the canonical representation of $N$ in $\ell^{2}(\operatorname{Spec}(N))$. Once this choice is done, the minimal projections of $M$ are just elements of $\mathbb{P}_{N}$. One can partially fix the gauge in the generic case by requiring that one of the elements of $\operatorname{Spec}_{N}(M)$ has all coordinates $>0$.

We shall now give an equivalent description of $\mathbb{P}_{N}$ which will allow one to treat the case where the action of $M$ has multiplicity $>1$. For each $k$, let $\mathbf{H}_{k}(N)$ be the space of rank $k$ positive hermitian matrices $\rho_{\lambda \mu}$ with labels $\lambda, \mu \in \operatorname{Spec}(N)$. One way to encode a point in $y \in \mathbb{P}_{N}$ is by the corresponding rank one matrix $\rho \in \mathbf{H}_{1}(N)$,

$$
\rho_{\lambda \mu}=\bar{z}_{\lambda} z_{\mu}, \quad z \in S_{\mathbb{C}}^{N}, p(z)=y
$$

We can then view $S=\operatorname{Spec}_{N}(M)$ as a subset with $n$ elements in $\mathbf{H}_{1}(N)$ such, for the matrix product, the following conditions hold:

- $\rho^{2}=\rho$ for all $\rho \in S$

- $\rho \rho^{\prime}=0$ for all $\rho \neq \rho^{\prime} \in S$

- $\sum_{S} \rho_{\lambda \mu}=\delta_{\lambda \mu}$

This is just encoding the partition of unity in the rank one minimal projections of $M$. In these coordinates the gauge action of the unitary group $\mathcal{U}(N)$ becomes the adjoint action:

$$
(\operatorname{Ad}(u) \rho)_{\lambda \mu}=u_{\lambda} \rho_{\lambda \mu} \bar{u}_{\mu}
$$

It might seem that replacing the subset $\operatorname{Spec}_{N}(M)=p(G(\pi)) \subset \mathbb{P}_{N}$ by the subset of $\mathbf{H}_{1}(N)$ is a useless complication but it allows one to extend the definition and properties of $\operatorname{Spec}_{N}(M)$ to the case when the abelian von Neumann algebra $M$ is no longer maximal abelian. We fix its multiplicity ${ }^{3}$ to be constant equal to $m$. We then get in the Hilbert space $\ell^{2}(\operatorname{Spec}(N))$ a partition of unity in the rank $m$ minimal projections of $M$. They form a subset with $n / m$ elements $S \subset \mathbf{H}_{m}(N)$ and fulfill exactly the same rules as above. We thus get:

\footnotetext{
${ }^{3}$ It can be easily extended to the general case of non-constant multiplicity.
} 
Proposition 2.6. Let $N$ be a maximal abelian von Neumann subalgebra of $\mathcal{L}(\mathcal{H})$ with $\mathcal{H}$ of dimension $n$. The relative position of abelian von Neumann algebras of constant multiplicity $m$ in $\mathcal{L}(\mathcal{H})$ is classified by subsets $S=\operatorname{Spec}_{N}(M) \subset \mathbf{H}_{m}(N)$ with $n / m$ elements such that (for the matrix product)

- $\rho^{2}=\rho$ for all $\rho \in S$

- $\rho \rho^{\prime}=0$ for all $\rho \neq \rho^{\prime} \in S$

- $\sum_{S} \rho_{\lambda \mu}=\delta_{\lambda \mu}$

The subset $S$ modulo the adjoint action of the unitary group $\mathcal{U}(N)$ is a complete invariant.

Proof. This is a simple reformulation of the equivalence between giving $M$ and giving the partition of unity by its minimal projections in the Hilbert space $\ell^{2}(\operatorname{Spec}(N))$ which is isomorphic to $\mathcal{H}$ with an isomorphism which is unique modulo the unitary group $\mathcal{U}(N)$.

\subsection{The CKM matrix and Fourier transform.}

Let us compute the above invariant $\operatorname{Spec}_{N}(M)$ in a simple example. We consider a finite abelian group $G$ and the pair of maximal abelian subalgebras of $\ell^{2}(G)$ given by the algebra $N=\ell^{\infty}(G)$ of multiplication operators and the algebra $M$ of convolution operators. We let $G=\mathbb{Z} / 3 \mathbb{Z}$ as a concrete example. We take the base points given for the algebra $N$ by the delta function at $1 \in G$ and for $M$ by the idempotent $e_{M}(g)=1$ of the convolution 4 algebra $C^{*}(G)$. The corresponding CKM matrix is of the form

$$
C=\frac{1}{\sqrt{3}}\left[\begin{array}{ccc}
1 & 1 & 1 \\
1 & e^{\frac{2 \pi i}{3}} & e^{-\frac{2 \pi i}{3}} \\
1 & e^{-\frac{2 \pi i}{3}} & e^{\frac{2 \pi i}{3}}
\end{array}\right]
$$

which gives with the notations of (3),

$$
c_{1}=\frac{1}{\sqrt{3}}, s_{1}=\sqrt{\frac{2}{3}}, c_{2}=s_{2}=\frac{1}{\sqrt{2}}, c_{3}=s_{3}=-\frac{1}{\sqrt{2}}, \quad e_{\delta}=i,
$$

The relative spectrum $\operatorname{Spec}_{N}(M)$ in the sense of Definition 2.5 is given by the three points in $\mathbb{P}_{2}(\mathbb{C})$ given by the lines of the above matrix. These three points are pairwise orthogonal and the gauge fixing corresponds to one of them (namely $(1,1,1))$ having all its coordinates positive. Note that $\operatorname{Spec}_{N}(M)$ is invariant under the complex conjugation but the CKM matrix itself is not, since $e_{\delta}=i$. This corresponds to the nuance with the more refined invariant of $M$ where the labeling of the spectrum is specified.

Remark 2.7. The above example falls in the special class of commuting squares (cf. [19]). More precisely ( $c f$. [19], Definition 2.2) a pair of von Neumann subalgebras $(M, N)$ of a finite von Neumann algebra $P$ with normalized trace $\tau$ is called mutually orthogonal when

$$
\tau\left(b_{1} b_{2}\right)=\tau\left(b_{1}\right) \tau\left(b_{2}\right), \quad \forall b_{1} \in M, b_{2} \in N .
$$

This condition is equivalent in the above context of a pair of maximal abelian von Neumann subalgebras $(M, N)$ of $M_{n}(\mathbb{C})$ to $\left|C_{x y}\right|=1 / \sqrt{n}$ for all $x, y$.

\footnotetext{
${ }^{4}$ We use the normalized Haar measure of total mass 1.
} 


\section{Relative Position of the CONTINUUm AND The DiscRete}

Let us first recall the following result of von Neumann ([21, 22]) which shows that there is a unique way to represent the continuum with constant multiplicity $m$.

Theorem 3.1. Let $\mathcal{H}$ be an infinite dimensional separabl 5 Hilbert space and $m$ an integer. There exists up to unitary equivalence only one commutative von Neumann subalgebra $M \subset \mathcal{L}(\mathcal{H})$ such that,

(1) $M$ contains no minimal projection,

(2) The commutant of $M$ is isomorphic to $M_{m}(M)$.

Proof. We briefly recall the proof of Theorem 3.1. The meaning of (1) is that $M$ represents the continuum, while the meaning of (2) is that the multiplicity of $M$ in $\mathcal{H}$ is equal to $m$. The separability of $\mathcal{H}$ shows that $M$ is generated 6 by a countable collection $T_{k}$ of commuting self-adjoint operators and hence by a single self-adjoint operator $T=f_{\infty}\left(T_{1}, \ldots, T_{k}, \ldots\right)$ where $f_{\infty}$ is a Borel injection of an infinite product of intervals in $\mathbb{R}$ into the real line $\mathbb{R}$ so that $T_{k}$ is a Borel function $f_{k}(T)$ of $T$ for each $k$. The multiplicity hypothesis shows that the representation of $M$ in $\mathcal{H}$ is the direct sum of $m$ copies of the representation in $e \mathcal{H}=\mathcal{H}_{1}, e$ a minimal projection in $M_{m}(\mathbb{C}) \subset M_{m}(M)$. Thus one can assume that $m=1$. In that case $M$ has a cyclic and separating unit vector $\xi$ and the spectral Theorem shows that the operator $T$ is unitarily equivalent to the operator of multiplication by $x$ in $\mathcal{H}_{1}=L^{2}(K, d \nu)$ where $K \subset \mathbb{R}$ is the compact spectrum of $T$ and $d \nu$ the spectral measure,

$$
\int f(x) d \nu(x)=\langle\xi, f(T) \xi\rangle .
$$

Since $M$ contains no minimal projection, the function $\nu(u)=\int_{-\infty}^{u} d \nu(x)$ is continuous. One has, for $u<v \in \mathbb{R}$,

$$
\nu(u) \leq \nu(v), \quad \nu(u)=\nu(v) \Leftrightarrow[u, v] \cap K=\emptyset
$$

Thus $\nu$ is injective a.e. for $d \nu$, the operator $\nu(T)$ generates $M$ and is unitarily equivalent to the operator of multiplication by $x$ in $L^{2}([0,1], d x)$. It follows that the pair $(M, \mathcal{H})$ is unitarily equivalent to the pair

$$
\left(L^{\infty}(X, \mu), L^{2}(X, \mu, S)\right), \quad X=[0,1], \mu=d x, S=\mathbb{C}^{m},
$$

where the trivial bundle with fibers $S_{x}=\mathbb{C}^{m}$ is viewed as a measurable hermitian vector bundle $S$ of dimension $m$ over $X$, and the action of $M=L^{\infty}(X, \mu)$ is given by multiplication in the Hilbert $L^{2}(X, \mu, S)$ of $L^{2}$-sections of $S$.

It is useful in general to keep the flexibility of describing the above model of the pair $(M, \mathcal{H})$ using (16) with $(X, \mu)$ a standard probability space and a measurable hermitian vector bundle $S$ of dimension $m$ over $X$. This is unique up to equivalence (21] §9 Definition 3).

Our goal in this section is to extend the result of $\$ 2$ and get a complete invariant of the relative position in $\mathcal{H}$ of the pair $(M, N)$ where $M$ is as in Theorem 3.1 and $N$ is a discrete maximal abelian von Neumann subalgebra $N \subset \mathcal{L}(\mathcal{H})$. By discrete we mean that $N$ is generated by its minimal projections. In that case it is isomorphic to $\ell^{\infty}(\mathbb{N})$ acting in $\ell^{2}(\mathbb{N})$ by multiplication. We shall later adapt the result to the case when $N$ has multiplicity and this will not introduce any new major difficulty.

\footnotetext{
${ }^{5}$ i.e. with countable orthonormal basis.

${ }^{6}$ As a von Neumann algebra.
} 


\subsection{The Frechet manifolds $\mathbf{H}_{m}(\Lambda), \mathbf{P}_{m}(\Lambda)$ and $\mathbf{G r}_{m}(\Lambda)$.}

We let $\Lambda$ be a countable set, which will label the set $\operatorname{Spec}(N)$ of minimal projections of the discrete von Neumann algebra $N$. Let $\mathbf{H}_{m}(\Lambda)$ be the space of positive hermitian forms of rank $m$ of the form $\rho_{\lambda \mu}$ where the indices $\lambda, \mu$ belong to $\Lambda$. Saying that $\rho_{\lambda \mu}$ is of rank $m$ means that the separated completion of the space $c_{c}(\Lambda)$ of sequences with finite support on $\Lambda$ for the inner product,

$$
\langle a, b\rangle=\sum \rho_{\lambda \mu} \bar{a}_{\lambda} b_{\mu}, \quad \forall a, b \in c_{c}(\Lambda)
$$

is a finite dimensional Hilbert space $\mathbf{S}(\rho)$ of dimension $m$. Note that the completion is unnecessary since a dense subspace of a finite dimensional Hilbert space is equal to the Hilbert space. Thus $\mathbf{S}(\rho)$ is the quotient

$$
\mathbf{S}(\rho)=c_{c}(\Lambda) / J_{\rho}, \quad J_{\rho}=\left\{a \in c_{c}(\Lambda) \mid \sum \rho_{\lambda \mu} \bar{a}_{\lambda} a_{\mu}=0\right\} .
$$

By construction the radical $J_{\rho}$ of $\rho$ is a codimension $m$ subspace of $c_{c}(\Lambda)$. It can be equivalently described by orthogonality with the lines of $\rho$ i.e.

$$
J_{\rho}=\left\{a \in c_{c}(\Lambda) \mid \sum \rho_{\lambda \mu} a_{\mu}=0, \quad \forall \lambda \in \Lambda\right\} .
$$

We let $\mathbf{G r}_{m}(\Lambda)$ be the Grassmanian of all codimension $m$ subspaces $J$ of $c_{c}(\Lambda)$. It is described equivalently as the Grassmanian of all $m$-dimensional subspaces $H$ of the Frechet space $\mathbb{C}^{\Lambda}$ using the canonical duality between $c_{c}(\Lambda)$ and $\mathbb{C}^{\Lambda}$ and the map $J \mapsto H=J^{\perp}$. We can use Grassmann coordinates

$$
H \mapsto \pi(H)=\xi_{1} \wedge \cdots \wedge \xi_{m}, \quad \forall\left(\xi_{j}\right) \text { basis of } H
$$

and view $\mathbf{G r}_{m}(\Lambda)$ as a subset of the projective space $\mathbb{P}\left(\wedge^{m} \mathbb{C}^{\Lambda}\right)$ over the linear space $\wedge^{m} \mathbb{C}^{\Lambda}$. There is a natural duality between $\wedge^{m} \mathbb{C}^{\Lambda}$ and $\wedge^{m} c_{c}(\Lambda)$ and we endow $\wedge^{m} \mathbb{C}^{\Lambda}$ with the weak topology coming from this duality. One can identify $\wedge^{m} c_{c}(\Lambda)$ with $c_{c}\left(\wedge^{m} \Lambda\right)$ where we let $\wedge^{m} \Lambda$ denote the set of oriented subsets $F \subset \Lambda$ with $m$ elements. The range of $\pi$ is characterized by the Plücker relations: $i_{V} P \wedge P=0$ for the contraction of $P=\pi(H)$ with any $V \in \wedge^{m-1} c_{c}(\Lambda)$. A local coordinate chart in $\mathbf{G r}_{m}(\Lambda)$ around $H$ is obtained by choosing a closed supplement $H^{\prime}$ of $H$ in $\mathbb{C}^{\Lambda}$, e.g. with $H=J^{\perp}$ by taking an $m$-dimensional supplement $J^{\prime}$ of $J$ in $c_{c}(\Lambda)$ and letting $H^{\prime}$ be the orthogonal of $J^{\prime}$. The domain of the local chart is the set of $m$-dimensional subspaces of $\mathbb{C}^{\Lambda}$ which intersect $H^{\prime}$ trivially. The local chart describes these subspaces as the graphs of arbitrary linear maps $T: H \rightarrow H^{\prime}$. Let $p\left(H, H^{\prime}\right)$ be the projection on $H$ parallel to $H^{\prime}$. The change of charts from $\left(H_{1}, H_{1}^{\prime}\right)$ to $\left(H_{2}, H_{2}^{\prime}\right)$ is given by

$$
T_{2}=\left(1+T_{1}\right) R\left(T_{1}\right)-1
$$

where $R\left(T_{1}\right)$ is the inverse of the map $\xi \in H_{1} \mapsto p\left(H_{2}, H_{2}^{\prime}\right)\left(\xi+T_{1} \xi\right) \in H_{2}$. Indeed for $\eta \in H_{2}$ one has $\eta+T_{2} \eta=\left(1+T_{1}\right) \xi$ for $\xi=R\left(T_{1}\right) \eta \in H_{1}$. Moreover $T_{2} \eta=$ $\left(1-p\left(H_{2}, H_{2}^{\prime}\right)\right)\left(1+T_{1}\right) \xi \in H_{2}^{\prime}$. By construction (21) only involves inverses of linear maps acting in finite dimensional spaces. Thus $\mathbf{G r}_{m}(\Lambda)$ is a Frechet manifold.

Proposition 3.2. 1) The quotient $\mathbf{S}(J)=c_{c}(\Lambda) / J$ yields a vector bundle $\mathbf{S}$ of dimension $m$ over $\mathbf{G r}_{m}(\Lambda)$.

2) The map $\rho \in \mathbf{H}_{m}(\Lambda) \mapsto j(\rho)=J_{\rho} \in \mathbf{G r}_{m}(\Lambda)$ is a locally trivial fibration with fiber the $m^{2}$-dimensional real open cone of non-degenerate positive hermitian forms on the corresponding fiber of the vector bundle $\mathbf{S}$. 
Proof. 1) On the domain of a local chart associated to the pair $\left(H, H^{\prime}\right)$ as above, the projection on $H$ parallel to $H^{\prime}$ gives a local trivialization of the bundle $\mathbf{S}$. The change of charts from $\left(H_{1}, H_{1}^{\prime}\right)$ to $\left(H_{2}, H_{2}^{\prime}\right)$ is given by $T_{1} \mapsto R\left(T_{1}\right)$ which is a smooth map to invertible linear maps from $H_{2}$ to $H_{1}$.

2) Let $J \subset c_{c}(\Lambda)$ be a subspace of codimension $m$ and $\mathbf{S}(J)=c_{c}(\Lambda) / J$. Giving $\rho \in \mathbf{H}_{m}(\Lambda)$ such that $j(\rho)=J$ is equivalent to choosing a non-degenerate positive hermitian form $h$ on $\mathbf{S}(J)$. One lets $\rho_{\lambda \kappa}=\left\langle\delta_{\lambda}, \delta_{\kappa}\right\rangle_{h}$ for all $\lambda, \kappa \in \Lambda$.

We denote by $\mathbf{P}_{m}(\Lambda)$ the quotient of $\mathbf{H}_{m}(\Lambda)$ by the scaling action of $\mathbb{R}_{+}^{*}$,

$$
\mathbf{P}_{m}(\Lambda)=\mathbf{H}_{m}(\Lambda) / \mathbb{R}_{+}^{*}, \quad \mathbb{R}_{+}^{*} \rightarrow \mathbf{H}_{m}(\Lambda) \stackrel{q}{\rightarrow} \mathbf{P}_{m}(\Lambda) .
$$

Proposition 3.3. 1) The scaling action of $\mathbb{R}_{+}^{*}$ turns $\mathbf{H}_{m}(\Lambda)$ into a principal $\mathbb{R}_{+}^{*}$ bundle $L$ over $\mathbf{P}_{m}(\Lambda)$.

3) A section $\sigma$ of the restriction of $L$ to $Y \subset \mathbf{P}_{m}(\Lambda)$ gives a hermitian metric on the restriction of $\mathbf{S}$ to $Y$ by

$$
\langle\xi, \eta\rangle_{y}=\sum \rho_{\mu \nu}(\sigma(y)) \bar{\xi}_{\mu} \eta_{\nu}, \quad \forall \xi, \eta \in c_{c}(\Lambda)
$$

Proof. The proof is straightforward using Proposition 3.2 .

\subsection{Injectivity of the map to hermitian forms.}

As explained above, there is, up to measurable isomorphism, a unique model of the inclusion $M \subset \mathcal{L}(\mathcal{H})$. It is given by a standard probability space $(X, \mu)$ and a measurable hermitian vector bundle $S$ of dimension $m$ over $X$, and the action of $M=L^{\infty}(X, \mu)$ by multiplication in the Hilbert $L^{2}(X, \mu, S)$ of $L^{2}$-sections of $S$.

Lemma 3.4. Let $\mathcal{H}$ be the Hilbert space $L^{2}(X, \mu, S)$ and $\left(\xi_{n}\right)_{n \in \mathbb{N}}$ an orthonormal basis. Then the map $\gamma$ which to $x \in X$ associates the matrix

$$
\gamma(x)=\gamma_{n, m}(x)=\left\langle\xi_{n}(x), \xi_{m}(x)\right\rangle
$$

is injective outside a null-set even after moding out by the scaling action of $\mathbb{R}_{+}^{*}$.

Proof. Let $\xi=\sum a_{n} \xi_{n} \in \mathcal{H}$. The sequence $\left(a_{n}\right)$ belongs to $\ell^{2}(\mathbb{N})$. Let $m_{k} \in \mathbb{N}$ be such that

$$
\sum_{m_{k}}^{\infty}\left|a_{n}\right|^{2} \leq 2^{-2 k} .
$$

One then has, with $\zeta_{k}=\sum_{1}^{m_{k}} a_{n} \xi_{n}$, that $\left\|\xi-\zeta_{k}\right\| \leq 2^{-k}$ and thus except on a subset $E_{k} \subset X$ of measure $\mu\left(E_{k}\right) \leq 2^{-k}$ one has $\left\|\xi(x)-\zeta_{k}(x)\right\|^{2} \leq 2^{-k}$. Thus by the Borel-Cantelli Lemma, for almost all $x \in X$ one has $x \notin E_{k}$ except for finitely many values of $k$ and hence $\zeta_{k}(x) \rightarrow \xi(x)$. This shows that for almost all $x \in X$

$$
\|\xi(x)\|^{2}=\lim _{k \rightarrow \infty} \sum_{n=1}^{m_{k}} \sum_{n^{\prime}=1}^{m_{k}} \bar{a}_{n} a_{n^{\prime}} \gamma_{n, n^{\prime}}(x) .
$$

In particular it shows that, for any two given vectors $\xi, \eta \in \mathcal{H}$, the knowledge of $\gamma_{n, m}(x)$ suffices to determine almost everywhere the inner product $\langle\xi(x), \eta(x)\rangle$. This shows that the problem of injectivity outside a null-set of the map $x \mapsto \gamma(x)$ is in fact independent of the choice of the orthonormal basis $\left(\xi_{n}\right)_{n \in \mathbb{N}}$. More precisely let $\left(\xi_{n}\right)_{n \in \mathbb{N}}$ be an orthonormal basis such that $q \circ \gamma$ is injective in the complement of the null set $X_{0} \subset X$. Let $\left(\xi_{n}^{\prime}\right)_{n \in \mathbb{N}}$ be another orthonormal basis. Then by the 
above argument one can express the matrix elements $\gamma_{n, m}(x)$ as a pointwise limit of linear functions

$$
\gamma_{n, m}(x)=\lim _{r} L_{n, m}^{(r)}\left(\gamma_{k, \ell}^{\prime}(x)\right)
$$

of the $\gamma_{k, \ell}^{\prime}(x)$ except on a null set $X_{1} \subset X$. It follows that the map $q \circ \gamma^{\prime}$ is injective except on the null set $X_{0} \cup X_{1}$ since the proportionality

$$
\gamma_{k, \ell}^{\prime}(x)=\lambda \gamma_{k, \ell}^{\prime}(y), \quad \forall k, \ell
$$

implies $\gamma_{n, m}(x)=\lambda \gamma_{n, m}(y)$ for all $n, m$. Up to isomorphism we can assume that the triple $(X, \mu, S)$ is given by the circle $X=S^{1}$ with the measure $d \theta$ and that the bundle $S$ is the trivial bundle. We first take the case where $S$ is of dimension one. Then we have $L^{2}(X, \mu, S)=L^{2}\left(S^{1}, d \theta\right)$ and we can choose the basis given by $\xi_{n}(\theta)=e^{i n \theta}$ labeled by $n \in \mathbb{Z}$. The map $\gamma$ is then

$$
\gamma_{n, m}(\theta)=e^{i(m-n) \theta}, \quad \forall m, n \in \mathbb{Z} .
$$

A relation of proportionality $\gamma(\theta)=\lambda \gamma\left(\theta^{\prime}\right)$ means

$$
e^{i(m-n) \theta}=\lambda e^{i(m-n) \theta^{\prime}}, \quad \forall m, n \in \mathbb{Z} .
$$

Taking $m=n$ gives $\lambda=1$ and then for $m=1, n=0$ one gets that $\theta=\theta^{\prime}$ (modulo $2 \pi$ ). With $S$ trivial of dimension $\ell$ we take a basis of the form $\xi_{n, k}(\theta)=e^{i n \theta} \epsilon_{k}$ where the $\epsilon_{k}$ form an orthonormal basis of $S$. One then has

$$
\gamma_{(n, k),(m, \ell)}(\theta)=e^{i(m-n) \theta} \delta_{k, \ell}
$$

and the map $\gamma$ is injective even after moding out by a scaling factor, since this already holds for the components $\gamma_{(n, 1),(m, 1)}$.

\subsection{The relative spectrum $\operatorname{Spec}_{N}(M)$.}

A discrete maximal abelian von Neumann subalgebra $N$ of $\mathcal{L}(\mathcal{H})$ is isomorphic to the algebra $\ell^{\infty}(\Lambda)$ acting by multiplication in $\ell^{2}(\Lambda)$ where $\Lambda=\operatorname{Spec}(N)$ is the countable set of minimal projections of $N$.

Let $F: L^{2}(X, \mu, S) \rightarrow \ell^{2}(\Lambda)$ be a unitary isomorphism. Let $\epsilon_{\lambda}, \lambda \in \Lambda$, be the canonical basis of $\ell^{2}(\Lambda)$. The vectors $\eta_{\lambda}=F^{*} \epsilon_{\lambda}$ form an orthonormal basis of $L^{2}(X, \mu, S)$. Each of them is a measurable $L^{2}$-section $\eta_{\lambda}(x)$ of $S$ on $X$ and is defined almost everywhere modulo $\mu$. Thus the following map is well defined almost everywhere modulo $\mu$,

$$
\varphi(x) \in \mathbf{H}_{m}(\Lambda), \quad \varphi(x)_{\lambda \kappa}=\left\langle\eta_{\lambda}(x), \eta_{\kappa}(x)\right\rangle
$$

Lemma 3.4 shows that this map is injective outside a null-set even after composition with the quotient map q, i.e. passing to $\mathbf{P}_{m}(\Lambda)$.

Definition 3.5. A measured section of the bundle $L$ over $\mathbf{P}_{m}(\Lambda)$ is an equivalence class of pairs $(\nu, \xi)$ of a positive finite measure $\nu$ on $\mathbf{P}_{m}(\Lambda)$ and a section $\xi$ of $L$ defined almost everywhere for $\nu$, modulo the equivalence relation

$$
(\nu, \xi) \sim\left(h \nu, h^{-1} \xi\right), \quad \forall h: \mathbf{P}_{m}(\Lambda) \rightarrow \mathbb{R}_{+}^{*} .
$$

We work as above in the measurable category, so that the function $h$ in (26) is a measurable function. 
Lemma 3.6. 1) The pair $(\nu, \xi)$ where

$$
\nu=(q \circ \varphi)_{*}(\mu), \quad \xi(q \circ \varphi(x))=\varphi(x)
$$

is a measured section of $L$.

2) The measured section of $L$ given by (27) only depends upon the von Neumann subalgebra $M=F L^{\infty}(X, \mu) F^{*} \subset \mathcal{L}\left(\ell^{2}(\Lambda)\right)$.

Proof. 1) By Lemma 3.4 the map $q \circ \varphi$ is injective and thus the equation $\xi(q \circ$ $\varphi(x))=\varphi(x)$ determines a section $\xi$ of $L$ on its range $Y=q \circ \varphi(X)$. Thus the pair $(\nu, \xi)$ is a measured section of $L$.

2) Let $X^{\prime}, \mu^{\prime}, S^{\prime}, F^{\prime}$ be such that, as for $F$, the unitary $F^{\prime}$ is an isomorphism $F^{\prime}$ : $L^{2}\left(X^{\prime}, \mu^{\prime}, S^{\prime}\right) \rightarrow \ell^{2}(\Lambda)$. Assume that, as subsets of $\mathcal{L}\left(\ell^{2}(\Lambda)\right)$,

$$
F L^{\infty}(X, \mu) F^{*}=F^{\prime} L^{\infty}\left(X^{\prime}, \mu^{\prime}\right) F^{* *} .
$$

Then the unitary $F^{*} F^{\prime}$ conjugates the von Neumann algebra $L^{\infty}\left(X^{\prime}, \mu^{\prime}\right)$ with $L^{\infty}(X, \mu)$. Thus (11] A-85, [18 Theorem 4.11.9) there exists a measurable isomorphism (outside null sets) $\psi: X \rightarrow X^{\prime}$, a measurable function $h: X \rightarrow \mathbb{R}_{+}^{*}$ and an isomorphism $V$ of measurable hermitian bundles $\psi^{*}\left(S^{\prime}\right) \sim S$ such that

$$
\psi_{*}(h \mu)=\mu^{\prime}, \quad F^{*} F^{\prime}(\eta)(x)=h(x)^{1 / 2} V(x) \eta(\psi(x)) .
$$

The isometric property of $U=F^{*} F^{\prime}$ relates to the unitarity of $V$ by

$$
\int_{X}\left\|F^{*} F^{\prime}(\eta)(x)\right\|^{2} d \mu(x)=\int_{X}\|\eta(\psi(x))\|^{2} h(x) d \mu(x)=\int_{X^{\prime}}\left\|\eta\left(x^{\prime}\right)\right\|^{2} d \mu^{\prime}\left(x^{\prime}\right) .
$$

One has, with $U=F^{*} F^{\prime}$,

$$
U f U^{*}=f \circ \psi, \quad \forall f \in L^{\infty}\left(X^{\prime}, \mu^{\prime}\right) .
$$

Let, as above, $\eta_{\lambda}=F^{*} \epsilon_{\lambda}$ and $\eta_{\lambda}^{\prime}=F^{\prime *} \epsilon_{\lambda}$. One has $\eta_{\lambda}=U \eta_{\lambda}^{\prime}$ and thus

$$
\eta_{\lambda}(x)=h(x)^{1 / 2} V(x) \eta_{\lambda}^{\prime}(\psi(x))
$$

which since $V(x)$ is unitary, gives

$$
\left\langle\eta_{\lambda}(x), \eta_{\kappa}(x)\right\rangle=h(x)\left\langle\eta_{\lambda}^{\prime}(\psi(x)), \eta_{\kappa}^{\prime}(\psi(x))\right\rangle
$$

so that we get, with $\varphi$ and $\varphi^{\prime}$ defined by (25),

$$
\varphi(x)=h(x) \varphi^{\prime}(\psi(x)), \quad \forall x \in X .
$$

This shows that $q \circ \varphi=q \circ \varphi^{\prime} \circ \psi$ and thus that $(q \circ \varphi)_{*} \mu=\left(q \circ \varphi^{\prime}\right)_{*} \psi_{*} \mu$. One has $\mu^{\prime}=h^{\prime} \psi_{*} \mu$ where $h^{\prime}\left(x^{\prime}\right)=h\left(\psi^{-1}\left(x^{\prime}\right)\right)$ for $x^{\prime} \in X^{\prime}$. The maps $q \circ \varphi$ and $q \circ \varphi^{\prime}$ are isomorphisms of $X$ and $X^{\prime}$ with the same subset $Y \subset \mathbf{P}_{m}(\Lambda)$. The equality

$$
k(y)=h\left((q \circ \varphi)^{-1}(y)\right)=h^{\prime}\left(\left(q \circ \varphi^{\prime}\right)^{-1}(y)\right), \quad \forall y \in Y
$$

defines a measurable map $k: Y \rightarrow \mathbb{R}_{+}^{*}$, and one has

$$
\left(q \circ \varphi^{\prime}\right)_{*} \mu^{\prime}=k(q \circ \varphi)_{*} \mu, \quad \xi^{\prime}=k^{-1} \xi
$$

which shows that the measured section of (27) is an invariant.

Definition 3.7. We define the relative spectrum of $M$ relative to $N$ as the measured section of $L$ :

$$
\operatorname{Spec}_{N}(M)=\left((q \circ \varphi)_{*}(\mu), \xi(y)=\varphi\left((q \circ \varphi)^{-1}(y)\right)\right.
$$

defined up to the gauge action of the unitary group $\mathcal{U}(N)$. 
Given a measured section $\nu$ of $L$, one can consider the following canonically associated data

- The Hilbert space $L^{2}(\nu)$ of $L^{2}$-sections of $\mathbf{S}$ on $\mathbf{P}_{m}(\Lambda)$ for the following inner product 7

$$
\left\langle s, s^{\prime}\right\rangle=\int\left\langle s(y), s^{\prime}(y)\right\rangle_{\xi(y)} d \mu(y)
$$

- The vectors $\kappa_{\lambda} \in L^{2}(\nu)$, for $\lambda \in \Lambda$, such that $\kappa_{\lambda}(g)$ is the class of $\delta_{\lambda}$ in $\mathbf{S}_{g}=c_{c}(\Lambda) / J_{g}$ where $\delta_{\lambda} \in c_{c}(\Lambda)$ is the delta function.

- The action of the algebra of bounded measurable functions on $\mathbf{P}_{m}(\Lambda)$ by multiplication in $L^{2}(\nu)$.

By construction this latter action yields a commutative von Neumann algebra $M_{\nu}$ of multiplicity $m$ in the Hilbert space $L^{2}(\nu)$. It is continuous iff the measure on $\mathbf{P}_{m}(\Lambda)$ associated to $\nu$ is diffuse i.e. such that the measure of any point is zero.

Definition 3.8. A measured section $\nu$ of $L$ is unitary iff the vectors $\kappa_{\lambda} \in L^{2}(\nu)$ form an orthonormal basis of $L^{2}(\nu)$.

In that case the basis $\left(\kappa_{\lambda}\right)$ gives an action of $N$ in $L^{2}(\nu)$ as the von Neumann algebra of diagonal operators. We call the corresponding pair of von Neumann algebras $\left(M_{\nu}, N\right)$ acting in $L^{2}(\nu)$ the canonical pair associated to $\nu$.

We can now extend Proposition 2.6 to the case at hand of two von Neumann algebras, a discrete and a continuous one.

Theorem 3.9. The relative spectrum $\nu=\operatorname{Spec}_{N}(M)$ is a complete invariant of the relative position of $M$ relative to $N$. It can be any diffuse measured section of the $\mathbb{R}_{+}^{*}$ bundle L fulfilling the unitarity condition.

Proof. By Lemma 3.6, the relative spectrum is an invariant of the relative position of $N$ and $M$. Indeed, given $N$ and $M$ acting in the same Hilbert space $\mathcal{H}$ one first constructs a unitary isomorphism $\mathcal{H} \sim \ell^{2}(\Lambda)$, uniquely determined up to the action of the the unitary group $\mathcal{U}(N)$. Then one uses Lemma 3.6 to get the measured section $\nu$ of $L$ which only depends upon the von Neumann subalgebra $M \subset \mathcal{L}\left(\ell^{2}(\Lambda)\right)$ and is thus unique up to the gauge action of the unitary group $\mathcal{U}(N)$. To show that one obtains a complete invariant, it is enough to show that the original pair is unitarily equivalent to the canonical pair $\left(M_{\nu}, N\right)$ acting in $L^{2}(\nu)$. We take as above a model of the inclusion $M \subset \mathcal{L}(\mathcal{H})$ given by the action of $L^{\infty}(X, \mu)$ by multiplication in the Hilbert $L^{2}(X, \mu, S)$ of $L^{2}$-sections of $S$. The original pair of von Neumann subalgebras $(M, N)$ of $\mathcal{L}(\mathcal{H})$ is unitarily equivalent to the pair $\left(F L^{\infty}(X, \mu) F^{*}, \ell^{\infty}(\Lambda)\right)$ acting in $\ell^{2}(\Lambda)$ where $F$ is a unitary isomorphism $F: L^{2}(X, \mu, S) \rightarrow \ell^{2}(\Lambda)$. The unitary $F$ gives us the orthonormal basis $\eta_{\lambda}=F^{*}\left(\epsilon_{\lambda}\right)$ and:

- The map $\varphi: X \rightarrow \mathbf{H}_{m}(\Lambda)$ of (25) with $q \circ \varphi$ injective8.

- An isomorphism $V$ of hermitian measurable bundles $V: \varphi^{*} \mathbf{S} \sim S$.

- A unitary isomorphism $W: L^{2}(\nu) \rightarrow L^{2}(X, \mu, S)$.

At a point $x \in X$ the isomorphism $V(x)$ is given by

$$
V(x)\left(\delta_{\lambda}\right)=\eta_{\lambda}(x), \quad \forall \lambda \in \Lambda .
$$

\footnotetext{
7 independent of the choice of the representative $(\mu, \xi)$ of $\nu$.
}

8 except on a null set. 
It is unitary by construction and yields a unitary isomorphism $W: L^{2}(\nu) \rightarrow$ $L^{2}(X, \mu, S)$ given by

$$
(W \zeta)(x)=V(x) \zeta(\varphi(x)), \quad \forall \zeta \in L^{2}(\nu), x \in X .
$$

For $f \in L^{\infty}(X, \mu)$ the operator $W^{*} f W$ acts in $L^{2}(\nu)$ as multiplication by the function $g$ which is arbitrary (say 0$)$ outside $Y=q \circ \varphi(X)$ and is such that $g(q \circ$ $\varphi(x))=f(x)$ for $x \in X$. Thus $W^{*} L^{\infty}(X, \mu) W$ is the von Neumann algebra of multiplication by bounded measurable functions on $\mathbf{P}_{m}(\Lambda)$. The orthonormal basis $W^{*} \eta_{\lambda}$ is the canonical basis of the sections $\kappa_{\lambda}$ corresponding to the $\delta_{\lambda}$, thus the unitary $W^{*}$ gives the unitary equivalence of the original pair $(M, N)$ with the model $\left(M_{\nu}, N\right)$ acting in $L^{2}(\nu)$, i.e. the pair canonically associated to the measured section $\nu$ of $L$ given by $\nu=\operatorname{Spec}_{N}(M)$. This shows that the relative spectrum is a complete invariant of the relative position of $M$ and $N$.

Conversely, given a diffuse unitary measured section $\nu$ of $L$, one checks that the relative spectrum of the pair $\left(M_{\nu}, N\right)$ acting in $L^{2}(\nu)$ is equal to $\nu$.

\subsection{Multiplicity for the discrete algebra.}

The above result extends to the case when the action of $N$ in $\mathcal{H}$ is no longer maximal abelian but has some finite multiplicity, i.e. we assume that each minimal idempotent $e_{\lambda} \in N$ has finite dimensional range $E_{\lambda}$ in $\mathcal{H}$. The quickest way to deal with multiplicity is to extend $N$ to a maximal abelian algebra $\tilde{N} \supset N$ and take the invariant $\operatorname{Spec}_{\tilde{N}}(M)$ but use instead of the gauge group $\mathcal{U}(\tilde{N})$ the larger group which is the unitary group $\mathcal{U}\left(N^{\prime}\right)$ of the commutant $N^{\prime}$ of $N$. The adjoint action of this group on the space $\mathbf{H}_{m}(\tilde{N})$ of positive hermitian forms $g_{\alpha \beta}$ with $\alpha, \beta \in \operatorname{Spec}(\tilde{N})$ is obtained using the natural projection $r: \operatorname{Spec}(\tilde{N}) \rightarrow \operatorname{Spec}(N)$ and identifying $\mathcal{U}\left(N^{\prime}\right)$ with the group of unitary matrices $u_{\alpha \beta}$ with $\alpha, \beta \in \operatorname{Spec}(\tilde{N})$ such that $u_{\alpha \beta}=0$ except when $r(\alpha)=r(\beta)$. One then has

$$
\operatorname{Ad}(u)(g)=u g u^{*}, \quad \forall g \in \mathbf{H}_{m}(\tilde{N}), u \in \mathcal{U}\left(N^{\prime}\right) .
$$

The relative $\operatorname{spectrum} \operatorname{Spec}_{N}(M)$ is then $\operatorname{Spec}_{\tilde{N}}(M)$ modulo the adjoint action (39) of $\mathcal{U}\left(N^{\prime}\right)$.

It is important however to give a more intrinsic definition. In general one is given for each $\lambda \in \operatorname{Spec}(N)$ a finite dimensional Hilbert space $E_{\lambda}$ which is the range of the corresponding minimal projection $e_{\lambda} \in N$. One replaces $c_{c}(\operatorname{Spec}(N))$ in (17) and (18) by the space $c_{c}(\operatorname{Spec}(N), E)$ of sections with finite support of the Hermitian bundle $E$. In the definition of $\mathbf{H}_{m}(N)$ the $g_{\lambda \mu}$ are no longer scalars but are operators

$$
g_{\lambda \mu}: E_{\mu} \rightarrow E_{\lambda}
$$

which gives meaning to the expression

$$
g_{\lambda \mu} \bar{a}_{\lambda} b_{\mu}=\left\langle a_{\lambda}, g_{\lambda \mu}\left(b_{\mu}\right)\right\rangle, \quad \forall a_{\lambda} \in E_{\lambda}, b_{\mu} \in E_{\mu} .
$$

One defines $\mathbf{P}_{m}(N)$ as in (22) i.e. as the quotient of $\mathbf{H}_{m}(N)$ by the scaling action of $\mathbb{R}_{+}^{*}$. Proposition 3.3 holds, with (23) replaced by

$$
\langle\xi, \eta\rangle_{y}=\sum\left\langle\xi_{\mu}, g_{\mu \nu}(\sigma(y)) \eta_{\nu}\right\rangle, \quad \forall \xi, \eta \in c_{c}(\operatorname{Spec}(N), E)
$$

The hermitian vector bundle $\mathbf{S}(g)$ is defined in the same way, as the quotient of $c_{c}(\operatorname{Spec}(N), E)$ by the radical $J_{g}$. To define the relative invariant $\operatorname{Spec}_{N}(M)$, one 
lets $F: L^{2}(X, \mu, S) \rightarrow \ell^{2}(\operatorname{Spec}(N), E)$ be a unitary isomorphism. One adapts (25) as follows

$$
\varphi(x) \in \mathbf{H}_{m}(N), \quad\left\langle\xi, \varphi(x)_{\lambda \kappa} \eta\right\rangle=\left\langle F^{*}(\xi)(x), F^{*}(\eta)(x)\right\rangle, \quad \forall \xi \in E_{\lambda}, \eta \in E_{\kappa} .
$$

Taking an orthonormal basis $\epsilon_{\alpha}=\epsilon_{\lambda, i}$ in each $E_{\lambda}$ one has

$$
\left\langle F^{*}\left(\epsilon_{\alpha}\right)(x), F^{*}\left(\epsilon_{\beta}\right)(x)\right\rangle=\left\langle\epsilon_{\lambda, i}, \varphi(x)_{\lambda \kappa} \epsilon_{\kappa, j}\right\rangle
$$

so that by Lemma 3.4 the map $q \circ \varphi$ is injective outside a null set. Definition 3.5 and Lemma 3.6 are unchanged with $\ell^{2}(\operatorname{Spec}(N), E)$ instead of $\ell^{2}(\operatorname{Spec}(N))$. The only change occurs for the gauge group in Definition 3.7 .

Definition 3.10. We define the relative spectrum of $M$ relative to $N$ as the measured section of $L$ :

$$
\operatorname{Spec}_{N}(M)=\left((q \circ \varphi)_{*}(\mu), \xi(y)=\varphi\left((q \circ \varphi)^{-1}(y)\right)\right.
$$

defined up to the gauge action of the unitary group of $\operatorname{End}_{N}(E)$.

In the Definition 3.8 of the unitarity of the measured section $\nu$ one uses instead of the vectors $\kappa_{\lambda}$ the natural linear maps $\kappa_{\lambda}: E_{\lambda} \rightarrow L^{2}(\nu)$ where for all $\xi \in E_{\lambda} \subset$ $c_{c}(\operatorname{Spec}(N), E)$ one lets

$$
\kappa_{\lambda}(\xi)_{g}=\text { class of } \xi \in c_{c}(\operatorname{Spec}(N), E) / J_{g}, \forall g \in \mathbf{P}_{m}(N) .
$$

Theorem 3.9 holds unchanged.

\section{The unitary (CKM) invariant of Riemannian manifolds}

To any compact oriented smooth Riemannian manifold $X$ with Riemannian metric $g$ we associate the following spectral triple $9(M, \mathcal{H}, D)$,

$$
M=L^{\infty}(X, d v), \quad \mathcal{H}=L^{2}\left(X, \wedge^{*}\right), \quad D=d+d^{*},
$$

so that $\mathcal{H}$ is the Hilbert space of square integrable differential forms (with complex coefficients) on which the algebra $L^{\infty}(X, d v)$ acts by multiplication operators, while $D=d+d^{*}$ is the signature operator. In the even dimensional case one uses the volume form (which uses the orientation) to define ( $c f$. [15]) the $\mathbb{Z} / 2$-grading $\gamma$ of the Hilbert space $\mathcal{H}$ while in the odd dimensional case one uses the corresponding operator $\gamma$ of square 1 to reduce the Hilbert space to the subspace where $\gamma=1$.

\subsection{Completeness of the invariant.}

Usually a spectral triple is given by restricting to the algebra of smooth functions, but in the above case the latter algebra can be recovered from it von Neumann algebra weak closure using the domains of powers of $D$ to define smoothness. More precisely:

Proposition 4.1. The signature spectral triple $(M, \mathcal{H}, D)$ uniquely determines the compact smooth Riemannian manifold $X$.

\footnotetext{
${ }^{9}$ where $v$ is the volume form on $X$

10 with suitable powers of $i$
} 
Proof. Let $X_{j}$ be two compact oriented smooth Riemannian manifold and $\left(M_{j}, \mathcal{H}_{j}, D_{j}\right)$ the associated triples as in (46). Let $U: \mathcal{H}_{1} \rightarrow \mathcal{H}_{2}$ be a unitary operator such that

$$
U M_{1} U^{*}=M_{2}, \quad U D_{1} U^{*}=D_{2}
$$

Let $\mathcal{H}_{j}^{\infty}=\cap_{n}$ Dom $D_{j}^{n}$ be the intersection of domains of powers of the self-adjoint unbounded operator $D_{j}$. Then the algebra $C^{\infty}\left(X_{j}\right)$ is the subalgebra of $M_{j}=$ $L^{\infty}\left(X_{j}, d v_{j}\right)$ given by

$$
\mathcal{A}_{j}=\left\{f \in M_{j} \mid f \mathcal{H}_{j}^{\infty} \subset \mathcal{H}_{j}^{\infty}\right\}
$$

as can be seen by applying $f$ to the constant 0 -form 1 to get the inclusion $\mathcal{A}_{j} \subset$ $C^{\infty}\left(X_{j}\right)$ while the other inclusion follows since elements of $\mathcal{H}_{j}^{\infty}$ are exactly the smooth forms. Thus one gets from (47) that

$$
U C^{\infty}\left(X_{1}\right) U^{*}=C^{\infty}\left(X_{2}\right)
$$

and there exists a diffeomorphism $\psi: X_{2} \rightarrow X_{1}$, such that

$$
U f U^{*}=f \circ \psi, \quad \forall f \in C^{\infty}\left(X_{1}\right) .
$$

The Riemannian metric $g$ is uniquely determined for instance by the equality

$$
[D, f]^{2} \in \mathcal{A},[D, f]^{2}=-g^{\mu \nu} \partial_{\mu} f \partial_{\nu} f, \quad \forall f=f^{*} \in \mathcal{A}
$$

Corollary 4.2. The pair given by the spectrum 11 of $D$ and the relative spectrum $\operatorname{Spec}_{N}(M)$, where $N$ is the von Neumann algebra of functions of $D$, uniquely determines the compact smooth Riemannian manifold $X$.

Proof. The knowledge of the spectrum of $D$ with the multiplicities gives the operator $D$ acting in the Hilbert space $\mathcal{H}$ and the knowledge of the relative spectrum $\operatorname{Spec}_{N}(M)$ gives, by Theorem 3.9. the von Neumann subalgebra $M \subset \mathcal{L}(\mathcal{H})$ and hence the triple $(M, \mathcal{H}, D)$ of Proposition 4.1 .

Remark 4.3. Proposition 4.1 has an analogue where one uses the scalar Laplacian $\Delta$ instead of the signature operator. To determine the metric one uses

$$
[[\Delta, f], g] \in \mathcal{A},[[\Delta, f], g]=-2 g^{\mu \nu} \partial_{\mu} f \partial_{\nu} g, \quad \forall f=f^{*}, g=g^{*} \in \mathcal{A}
$$

In fact one can also use the embedding results of [2] which show how to recover the metric at the local level from the heat expansion. One definite advantage in using the Laplacian is that the eigenfunctions can be chosen to be real valued which further reduces the gauge group to the unitary group of the self-adjoint real subalgebra $N_{\mathrm{sa}}=\left\{x \in N \mid x=x^{*}\right\}$. Order one operators are however easier to characterize than order two operators, in particular for the orientability condition as we shall see below in 4.3 .

\footnotetext{
$11_{\text {with }}$ multiplicities
} 


\subsection{The spectral meaning of points.}

Once we know the spectrum $12 \Lambda$ of $D$, the missing information contained in $\operatorname{Spec}_{N}(M)$ is a measured section $\nu$ of the $\mathbb{R}_{+}^{*}$ bundle $L$ over $\mathbf{P}_{m}(\Lambda)$ fulfilling the unitarity condition. It should be interpreted as giving the probability for correlations between the possible frequencies, while a "point" of the geometric space $X$ can be thought of as a correlation, i.e. a specific positive hermitian matrix $g_{\lambda \kappa}$ (up to scale) in the support of $\nu$. To go further in this "spectral" identification of points one needs to check the injectivity of the map $\varphi$ of (25) at the topological rather than at the measure theoretic level of Lemma 3.4

Lemma 4.4. Let $X$ be a compact oriented smooth Riemannian manifold and $(M, \mathcal{H}, D)$ the spectral triple of (46). Then the map $\varphi$ of (25) is injective.

Proof. By construction one has

$$
\varphi(x) \in \mathbf{H}_{m}(N), \quad \varphi(x)_{\lambda \kappa}=\left\langle\eta_{\lambda}(x), \eta_{\kappa}(x)\right\rangle
$$

where the $\eta_{\lambda}$ form an orthonormal basis of eigenfunctions for $D$. The space $C^{\infty}(X, S)$ of smooth sections of the hermitian vector bundle $S$ over $X$ on which $D$ is acting coincides with the intersection of the domains of powers of $D$ and hence with the following vector space

$$
C^{\infty}(X, S)=\left\{\sum a_{\lambda} \eta_{\lambda} \mid a \in \mathcal{S}\right\}
$$

where $\mathcal{S}$ is the Schwartz space of sequences $\left(a_{\lambda}\right)$ of rapid decay. For any pair $\alpha=\sum a_{\lambda} \eta_{\lambda}, \beta=\sum b_{\lambda} \eta_{\lambda}$ of elements of $C^{\infty}(X, S)$, one has

$$
\langle\alpha(x), \beta(x)\rangle=\sum \bar{a}_{\lambda} b_{\kappa} \varphi(x)_{\lambda \kappa}, \quad \forall x \in X,
$$

where convergence can be checked using Sobolev estimates. Since any smooth function $f \in C^{\infty}(X)$ can be written in the form $f(x)=\langle\alpha(x), \beta(x)\rangle$ one gets the required injectivity of $\varphi$.

\subsection{The characterization of the range.}

The really difficult problem, then, is to characterize which values of these invariants correspond to compact smooth Riemannian manifolds. We shall only deal below with the case of $\operatorname{spin}^{c}$ manifolds in which case we use the Dirac spectral triple instead of the above signature triple.

The key result that we shall us is that under the simple conditions of [8] on a spectral triple $(\mathcal{A}, \mathcal{H}, D)$, with $\mathcal{A}$ commutative, the algebra $\mathcal{A}$ is the algebra $C^{\infty}(X)$ of smooth functions on a (unique) smooth compact manifold $X$. The five conditions ([8), in dimension $p$, are

(1) The $n$-th characteristic value of the resolvent of $D$ is $O\left(n^{-1 / p}\right)$.

(2) $[[D, a], b]=0 \quad \forall a, b \in \mathcal{A}$.

(3) For any $a \in \mathcal{A}$ both $a$ and $[D, a]$ belong to the domain of $\delta^{m}$, for any integer $m$ where $\delta$ is the derivation: $\delta(T)=[|D|, T]$.

\footnotetext{
12 with multiplicities
} 
(4) There exists $c \in \mathcal{A}^{\otimes n}, n=p+1$, totally antisymmetric in its last $p$-entries, and such that 13 $\pi_{D}(c)=1$, where $\pi_{D}\left(a_{0} \otimes \cdots \otimes a_{p}\right)=a_{0}\left[D, a_{1}\right] \cdots\left[D, a_{p}\right], \quad \forall a_{j} \in \mathcal{A}$.

(5) Viewed as an $\mathcal{A}$-module the space $\mathcal{H}_{\infty}=\cap \operatorname{Dom} D^{m}$ is finite and projective. Moreover the following equality defines a hermitian structure $(\mid)$ on this module: $\langle\xi, a \eta\rangle=f a(\xi \mid \eta)|D|^{-p}, \forall a \in \mathcal{A}, \forall \xi, \eta \in \mathcal{H}_{\infty}$.

In the last equation, $f$ is the noncommutative integral given by the Dixmier trace.

We can now restate Theorem 11.5 of [10] as:

Theorem 4.5. Let $(\mathcal{A}, \mathcal{H}, D)$ fulfill the above five conditions and assume that the multiplicity is $m=2^{p / 2}$, then there exists a unique smooth compact oriented spin ${ }^{c}$ Riemannian manifold $(X, g)$ such that the triple $(\mathcal{A}, \mathcal{H}, D)$ is given by

- $\mathcal{A}=C^{\infty}(X)$.

- $\mathcal{H}=L^{2}(X, S)$ where $S$ is the spinor bundle.

- $D$ is a Dirac operator associated to the Riemannian metric $g$.

Note that there is no uniqueness of $D$ since we only know its principal symbol. This is discussed in 8 and 13 . The Hochschild cycle $c$ gives the orientation.

Let us start with the spectrum $\Lambda=\operatorname{Spec}(D)$ given as a subset of $\mathbb{R}$ with multiplicity and condition (1) determines the growth of $\Lambda$. This fixes the Hilbert space $\mathcal{H}=$ $\ell^{2}(\Lambda)$ and the self-adjoint operator $D$ which is just a multiplication operator. The analogue in our context of the geodesic flow is the following one parameter group

$$
\gamma_{t}(T)=e^{i t|D|} T e^{-i t|D|}, \quad \forall T \in \mathcal{L}(\mathcal{H}) .
$$

Definition 4.6. We say that an operator $T \in \mathcal{L}(\mathcal{H})$ is of class $C^{\infty}$ when the map from $\mathbb{R}$ to $\mathcal{L}(\mathcal{H})$ given by $t \mapsto \gamma_{t}(T)$ is of class $C^{\infty}$ (for the norm topology of $\mathcal{L}(\mathcal{H})$ ) and we denote by $C^{\infty}(\mathcal{H}, D)$ this subalgebra of $\mathcal{L}(\mathcal{H})$.

This algebra only depends upon $(\mathcal{H}, D)$.

Definition 4.7. A unitary measured section $\nu$ of $L$ is smooth when

- The support $K$ of $\nu$ is compact.

- The map $a \in \mathcal{S}(\Lambda) \mapsto \sum a_{\lambda} \kappa_{\lambda}$ is an isomorphism of the Schwartz space $\mathcal{S}(\Lambda)$ with $C^{\infty}(K, \mathbf{S})$

- Any element of $C^{\infty}(K)$ is of class $C^{\infty}$ in the sense of Definition 4.6 .

Let then $\nu$ be a smooth unitary measured section of $L$. The order one condition (2) means that $D$ is a differential operator of order one. The regularity condition (3) is now automatically fulfilled. The orientability condition (4) can be formulated as the existence of a $p$-form $c$ on $K$ such that (55) holds. Finally condition (5) now becomes an equation.

\footnotetext{
${ }^{13}$ This assumes $p$ odd, in the even case one requires that $\pi_{D}(c)=\gamma$ fulfills $\gamma=\gamma^{*}, \gamma^{2}=$ $1, \gamma D=-D \gamma$.
} 


\section{The Sunada EXamples}

We have not gone very far in computing examples. The case of flat tori is straightforward, the only difficulty being to properly take care of the gauge ambiguity coming from the multiplicity of eigenvalues. Thus, instead, we shall concentrate on the examples of isospectral non-isometric Riemannian manifolds constructed by Sunada in 23. Let, as in [23, $G$ be a finite group and $H_{1}, H_{2}$ be subgroups of $G$ such that each conjugacy class $[g] \in[G]$ meets $H_{1}$ and $H_{2}$ in the same number of elements. The existence of examples where the $H_{j}$ are non-isomorphic was used in 23. to produce classes of examples of isospectral but non-isomorphic Riemannian geometries. One considers a compact oriented smooth Riemannian manifold $X$ on which the group $G$ acts freely by isometries. One then takes the quotient Riemannian manifolds $X_{j}=H_{j} \backslash X$. For each $y \in Y=G \backslash X$ the fiber $p^{-1}(y)$ of the projection $p: X \rightarrow Y$ can be identified with $G$ as a $G$-space for the left action of $G$ but there is a non-canonical choice of base point in the fiber. Two different choices are related by the right action of $G$. The essence of the situation is captured by the comparison, commuting with the right representation $\rho$ of $G$, of the von Neumann algebras $M_{j}$ of multiplication by functions on $H_{j} \backslash G$ in the Hilbert space $\ell^{2}\left(H_{j} \backslash G\right)$. The algebra that plays the role of the algebra of functions of $D$ is a subalgebra of the center of the convolution algebra $C^{*}(G)$. It acts on $\ell^{2}\left(H_{j} \backslash G\right)$ by right convolution which commutes with the projections $P_{j}: \ell^{2}(G) \rightarrow \ell^{2}\left(H_{j} \backslash G\right)$ given by averaging over $H_{j}$ i.e.

$$
P_{j}(\xi)(g)=\left(\# H_{j}\right)^{-1} \sum_{H_{j}} \xi(h g)
$$

By Lemma 2 of 23 the representations $\pi_{j}$ by right convolution of $C^{*}(G)$ in the Hilbert spaces $\mathcal{H}_{j}=\ell^{2}\left(H_{j} \backslash G\right)$ are equivalent since their characters are the same. Moreover this character is given by

$$
\operatorname{tr}\left(\pi_{j}(g)\right)=\operatorname{tr}\left(\rho(g) P_{j}\right)=\left(\# H_{j}\right)^{-1} \sum_{[k] \in[G]} \#\left([k] \cap H_{j}\right) \operatorname{tr}(\lambda(k) \rho(g))
$$

where $\lambda$ is the left regular representation of $G$ and $\rho$ the right regular one. On the canonical basis $\epsilon_{h}, h \in G$ of $\ell^{2}(G)$, the operator $\lambda(k) \rho(g)$ is a permutation and its trace is the number of fixed points, i.e. the cardinality of $\left\{x \in G \mid \mathrm{kxg}^{-1}=x\right\}$. It is non-zero when the conjugacy class of $k$ is the same as that of $g$ and is equal, in that case, to the order of the centralizer $C_{g}$. The latter is $\# C_{g}=\# G / \#[g]$ so that one gets

$$
\operatorname{tr}\left(\pi_{j}(g)\right)=(\# G)\left(\# H_{j}\right)^{-1}\left(\#\left([g] \cap H_{j}\right)\right)(\#[g])^{-1}
$$

The two projections $P_{j}$ are equivalent in the von Neumann algebra $\lambda(G)$ of the left-regular representation of $G$. One looks in fact for a unitary $U$ such that

$$
U \in \lambda(G), \quad U U^{*}=U^{*} U=1, \quad U P_{1} U^{*}=P_{2}, \quad U M_{1} U^{*}=M_{2}
$$

where $M_{j}$ is the algebra of multiplication by functions on $H_{j} \backslash G$. Since the two projections $P_{j}$ are equivalent in $\lambda(G)$ one can find a unitary $U_{0} \in \lambda(G)$ which fulfills all but the last conditions in (59). The remaining freedom is a unitary $V$ in the reduced algebra $P_{1} \lambda(G) P_{1}$. One looks for $V$ such that

$$
V \in P_{1} \lambda(G) P_{1}, \quad V V^{*}=V^{*} V=P_{1}, \quad V M_{1} V^{*}=U_{0}^{*} M_{2} U_{0} .
$$


We let $N$ be the quotient of $C^{*}(G)$ by the kernel of the representation $\pi_{j}$ and identify it with its image under $\pi_{j}$. Then we are comparing the two pairs $\left(M_{j}, N\right)$ as in Proposition 2.6 with $N$ no longer commutative.

Proposition 5.1. The two pairs $\left(M_{j}, N\right)$ are conjugate iff the subgroups $H_{j} \subset G$ are conjugate.

Proof. The data $\left(M_{j}, N\right)$ with $N=C^{*}(G) / \operatorname{Ker} \pi_{j}$, gives the set $\operatorname{Spec}\left(M_{j}\right)$ together with the action of $G$ on this set. Thus it gives back the $G$-space $H_{j} \backslash G$. This in turns determines uniquely the conjugacy class of the subgroup $H_{j} \subset G$ from the isotropy group of any point. Conversely an isomorphism of the $G$-spaces $H_{j} \backslash G$ yields an isomorphism of the $\left(M_{j}, N\right)$.

More generally, one can determine the behavior of the relative spectrum under the action of a finite group $H$ of automorphisms. We let $H$ be a finite group unitarily represented in the Hilbert space $\mathcal{H}$ of a spectral triple $(M, \mathcal{H}, D)$ so that

$$
\pi(h) D=D \pi(h), \quad \pi(h) M \pi(h)^{*}=M, \quad \forall h \in H .
$$

The spectral triple $\left(M_{H}, \mathcal{H}_{H}, D_{H}\right)$ is obtained as follows

$$
M_{H}=M \cap \pi(H)^{\prime}, \mathcal{H}_{H}=\{\xi \in \mathcal{H} \mid \pi(h) \xi=\xi, \forall h \in H\}, D_{H}=\left.D\right|_{\mathcal{H}_{H}} .
$$

so that the von Neumann algebra $M_{H}$ is the fixed point algebra under the action of $H$,

$$
M_{H}=\left\{x \in M \mid \pi(h) x \pi(h)^{*}=x, \forall h \in H\right\} .
$$

For each $\lambda \in \operatorname{Spec}(D)$ one lets $E_{\lambda} \subset \mathcal{H}$ be the corresponding eigenspace. Then let $\pi_{\lambda}$ be the restriction of the representation $\pi$ to $E_{\lambda}$. The eigenspace $F_{\lambda}$ of the spectral triple $\left(M_{H}, \mathcal{H}_{H}, D_{H}\right)$ is obtained as follows

$$
F_{\lambda}=E_{\lambda}^{H}=\left\{\xi \in E_{\lambda} \mid \pi(h) \xi=\xi, \forall h \in H\right\}
$$

We denote by $P_{\lambda}$ the orthogonal projection from $E_{\lambda}$ to $F_{\lambda}$. In order to compute the relative spectrum of $M_{H}$ relative to the algebra $N_{H}$ of functions of $D_{H}$ we use the intrinsic formulation of 3.4 on multiplicity.

Lemma 5.2. Assume that the action of $H$ on $M$ is free (i.e. the set of fixed points $x \in X, \exists h \neq 1, h x=x$ is negligible). The following defines a map from $\operatorname{Spec}_{N}(M) \subset \mathbf{H}_{m}(N)$ to $\mathbf{H}_{m}\left(N_{H}\right)$

$$
p(g)_{\lambda \kappa}=P_{\lambda} g_{\lambda \kappa} P_{\kappa}
$$

The map $p$ is invariant under the adjoint action of $H$ and $\operatorname{Spec}_{N_{H}}\left(M_{H}\right)$ is the image of $\operatorname{Spec}_{N}(M)$ under $p$.

We leave the proof as an exercise. We shall now show an explicit example where the two pairs $\left(M_{j}, N\right)$ of Proposition 5.1 are not conjugate but become so up to an automorphism of $N$. We use a concrete example of triple $\left(G, H_{1}, H_{2}\right)$ obtained as follows ( $c f$. 23]). One takes for $G$ the semi-direct product of the additive group $\mathbb{Z} / 8 \mathbb{Z}$ by the action of the multiplicative group $(\mathbb{Z} / 8 \mathbb{Z})^{*}=\{1,3,5,7\}$. It is a group of order 32 with multiplication given by

$$
(a, b) \cdot(c, d)=(a c, b+a d) \in(\mathbb{Z} / 8 \mathbb{Z})^{*} \times \mathbb{Z} / 8 \mathbb{Z}
$$

The adjoint action $\operatorname{Ad}(g)$ of $G$ on itself is given by

$$
\operatorname{Ad}(g)(x, y)=(x, a y+(1-x) b), \quad \forall g=(a, b) \in G
$$


The conjugacy classes $[g] \in[G]$ are the following

$$
\begin{gathered}
\{(1,0)\},\{(1,4)\},\{(1,2),(1,6)\},\{(1,1),(1,3),(1,5),(1,7)\}, \\
\{(3,0),(3,2),(3,4),(3,6)\},\{(3,1),(3,3),(3,5),(3,7)\} \\
\{(5,0),(5,4)\},\{(5,2),(5,6)\},\{(5,1),(5,3),(5,5),(5,7)\} \\
\{(7,0),(7,2),(7,4),(7,6)\},\{(7,1),(7,3),(7,5),(7,7)\}
\end{gathered}
$$

The subgroup $H_{1}=\left\{(a, 0) \mid a \in(\mathbb{Z} / 8 \mathbb{Z})^{*}\right\}$ has the same cardinality of intersection with the conjugacy classes of $G$ as the subgroup

$$
H_{2}=\{(1,0),(3,4),(5,4),(7,0)\}
$$

The character of the representation of $G$ given by (58) is the central function which vanishes except on the 4 conjugacy classes which meet the $H_{j}$. The characters of irreducible representations of $G$ are given from the orbits of the action of $(\mathbb{Z} / 8 \mathbb{Z})^{*}$ on the dual of $\mathbb{Z} / 8 \mathbb{Z}$ which we identify with $\mathbb{Z} / 8 \mathbb{Z}$ using the basic character $n \in$ $\mathbb{Z} / 8 \mathbb{Z} \mapsto \chi(n)=\omega^{n}$ where $\omega=e^{i \pi / 4}$ is a primitive 8 -th root of 1 . Thus each $n \in \mathbb{Z} / 8 \mathbb{Z}$ gives the character $m \mapsto \chi(n m)$ of $\mathbb{Z} / 8 \mathbb{Z}$. The eleven characters of $G$ $\operatorname{are} 14$

0 Four 1-dimensional characters $(a, b) \mapsto \kappa(a)$ where $\kappa$ is a character of $(\mathbb{Z} / 8 \mathbb{Z})^{*}$.

4 Four 1-dimensional characters $(a, b) \mapsto \kappa(a) \chi(4 b)$ where $\kappa$ is a character of $(\mathbb{Z} / 8 \mathbb{Z})^{*}$.

2 Two 2-dimensional characters given by

$$
\begin{gathered}
c_{2}((a, b))= \begin{cases}0 & \text { if } \quad a \notin\{1,5\} \text { or } b \notin 2 \mathbb{Z} \\
(-1)^{b / 2} 2 & \text { if } a \in\{1,5\}, b \in 2 \mathbb{Z} .\end{cases} \\
c_{2}^{\prime}((a, b))= \begin{cases}0 & \text { if } a \notin\{1,5\} \text { or } b \notin 2 \mathbb{Z} \\
(-1)^{b / 2+(a-1) / 4} 2 & \text { if } a \in\{1,5\}, b \in 2 \mathbb{Z} .\end{cases}
\end{gathered}
$$

1 One 4-dimensional character given by

$$
c_{4}((a, b))= \begin{cases}0 & \text { if } a \neq 1 \text { or } b \notin 4 \mathbb{Z} \\ (-1)^{b / 4} 4 & \text { if } a=1, b \in 4 \mathbb{Z} .\end{cases}
$$

The idempotent $4 c_{4}$ is the sum of the following 4 minimal projections $e_{m} \in C^{*}(G)$, for $m \in\{1,3,5,7\}$,

$$
e_{m}((a, b))= \begin{cases}0 & \text { if } a \neq 1 \\ 4 \chi(m b) & \text { if } a=1\end{cases}
$$

The 8-dimensional representation $\pi_{j}$ of $G$ in $\ell^{2}\left(H_{j} \backslash G\right)$ decomposes as the direct sum of 4 irreducible representations, each of multiplicity one, corresponding to the trivial character, the character $(a, b) \mapsto \chi(4 b)$ and the characters $c_{2}$ and $c_{4}$. We now identify the coset spaces $H_{j} \backslash G$ with $\mathbb{Z} / 8 \mathbb{Z}$, just as sets, to keep track of the algebras $M_{j}$ of multiplication operators. It is simpler for the right coset spaces $G / H_{j}$. By (65) the map $p_{1}(a, b)=b$ gives a bijection $G / H_{1} \rightarrow \mathbb{Z} / 8 \mathbb{Z}$. Similarly the bijection $p_{2}: G / H_{2} \rightarrow \mathbb{Z} / 8 \mathbb{Z}$ is obtained by intersecting each class with the subgroup

$$
L=\{(1, b) \mid b \in \mathbb{Z} / 8 \mathbb{Z}\} \subset G
$$

\footnotetext{
${ }^{14}$ With the label of the orbit of $(\mathbb{Z} / 8 \mathbb{Z})^{*}$ on $\mathbb{Z} / 8 \mathbb{Z}$.

${ }^{15}$ The Haar measure on $G$ is normalized of total mass 1
} 
which is transverse to the subgroups $H_{j}$. We use the identification $H_{j} \backslash G \sim L$ for the left coset spaces and thus obtain two representations $\pi_{j}$ of $G$ in $\ell^{2}(L)$.

Lemma 5.3. The von Neumann algebras $\pi_{j}(G)^{\prime \prime}$ are the same and the representations $\pi_{j}$ are inner related as follows

$$
\pi_{2}(g)=\pi_{1}\left(U g U^{*}\right), \quad \forall g \in G
$$

where $U \in C^{*}(G)$ is the unitary

$$
U=1-2\left(e_{3}+e_{5}\right)
$$

Proof. The character of the representation $\pi_{j}$ of $G$ in $\ell^{2}\left(H_{j} \backslash G\right)$ is the central function $\tau(g)$ which assigns to $g \in G$ the number of fixed points of right translation by $g$ in $H_{j} \backslash G$, it is given by (58). Thus the inner product of $\tau$ with the character $\alpha$ of a representation of $G$ is proportional to $\sum_{H_{j}} \alpha(g)$. Thus to know which representations of $G$ appear in $\pi_{j}$, one just computes $\sum_{H_{j}} \alpha(g)$ for the irreducible characters $\alpha$ listed above. It is enough to do it for $H_{1}=(\mathbb{Z} / 8 \mathbb{Z})^{*}$. One gets a non-zero result only for the trivial character, the character $(a, b) \mapsto \chi(4 b)$ and the characters $c_{2}$ and $c_{4}$. Since the dimension of $\ell^{2}\left(H_{j} \backslash G\right)$ is 8 the multiplicities are all equal to 1 . We decompose the idempotent $2 c_{2}$ as the sum of the following 2 minimal projections $e_{m} \in C^{*}(G)$, for $m \in\{2,6\}$,

$$
e_{m}((a, b))= \begin{cases}0 & \text { if } a \notin\{1,5\} \\ 2 \chi(m b) & \text { if } a \in\{1,5\} .\end{cases}
$$

We extend the notation for $m \in\{0,4\}$ using the characters of one dimensional irreducible representations

$$
e_{m}((a, b))=\chi(m b), \quad \forall m \in\{0,4\} .
$$

By construction the $\pi_{j}\left(e_{m}\right)$ are the minimal projections of a maximal abelian algebra acting in $\ell^{2}\left(H_{j} \backslash G\right)$. One checks that under the isomorphism $\ell^{2}\left(H_{1} \backslash G\right) \sim$ $\ell^{2}(L) \sim \ell^{2}\left(H_{2} \backslash G\right)$ one gets $\pi_{1}\left(e_{m}\right)=\pi_{2}\left(e_{m}\right)$ for all $m$. In fact it is enough to show that with $\sigma=\sum \chi(m) e_{m}$ the operator $\pi_{j}(\sigma)$ is the translation of 1 in $\ell^{2}(L)$. One finds (with Haar measure normalized as a probability measure) that

$$
\left(\pi_{j}(\sigma) f\right)(b)=f(b+1), \quad \forall f \in \ell^{2}(L), b \in L \sim \mathbb{Z} / 8 \mathbb{Z} .
$$

It follows from the equality $\pi_{1}\left(e_{m}\right)=\pi_{2}\left(e_{m}\right)$ for all $m$ that the von Neumann algebras $\pi_{j}(G)^{\prime \prime}$ are the same, since the partial isometry realizing the equivalence of minimal projections is unique up to a phase. One checks (171) by direct calculation.

We let the two pairs $\left(M_{j}, N\right)$ be as in Proposition 5.1

Proposition 5.4. Let $G$ and $H_{j} \subset G$ be as above then the following two pairs of von Neumann algebras are conjugate

$$
\left(M_{1}, \pi_{1}(G)^{\prime \prime}\right) \sim\left(M_{2}, \pi_{2}(G)^{\prime \prime}\right), M_{j}=\ell^{\infty}\left(H_{j} \backslash G\right)
$$

Any equivalence (76) induces a non-trivial automorphism of $N=C^{*}(G) / \operatorname{Ker} \pi_{j}$.

Proof. The isomorphism $\ell^{2}\left(H_{1} \backslash G\right) \sim \ell^{2}(L) \sim \ell^{2}\left(H_{2} \backslash G\right)$ transforms $M_{1}$ into $M_{2}$ and preserves the von Neumann algebras $\pi_{j}(G)^{\prime \prime}$ thus it induces the equivalence 
(76). This proves the first statement. To get the second it is enough to show that one cannot find a unitary $V$ acting in $\ell^{2}\left(H_{1} \backslash G\right)$ and such that

$$
V \ell^{\infty}\left(H_{1} \backslash G\right) V^{*}=\ell^{\infty}\left(H_{1} \backslash G\right), \quad V \pi_{1}(g) V^{*}=\pi_{1}\left(U g U^{*}\right), \quad \forall g \in G
$$

It follows that $V=\pi_{1}(U) Z$ where $Z$ is a unitary in the commutant of $\pi_{1}(G)$. This commutant is the following algebra

$$
C=\left\{\lambda_{0} e_{0}+\lambda_{4} e_{4}+2 \lambda c_{2}+4 \mu c_{4} \mid \lambda_{j}, \lambda, \mu \in \mathbb{C}\right\}
$$

Now the normalizer of $M_{1}=\ell^{\infty}\left(H_{1} \backslash G\right)$ intersected with the maximal abelian algebra generated by the $e_{m}$ is, up to a scalar factor of modulus one, the group of order 8 of translations generated by $\pi_{1}(\sigma)$. Thus one checks that for no unitary element $Z$ the product $V=\pi_{1}(U) Z$ is in the normalizer of $M_{1}=\ell^{\infty}\left(H_{1} \backslash G\right)$.

\section{REFERENCES}

[1] L. Autonne, Sur les matrices hypohermitiennes et les unitaires, Comptes Rendus de l'Académie des Sciences, Paris, vol. 156 (1913), 858-860.

[2] P. Bérard, G. Besson, S. Gallot Embedding Riemannian manifolds by their heat kernel, Geometric And Functional Analysis, Volume 4, Number 4 (1994), 373-398.

[3] E. T. Browne, The characteritic roots of a matrix, Bull. Amer. Math. Soc. vol. 36 (1930), $705-710$.

[4] A. Chamseddine, A. Connes, The Spectral action principle, Comm. Math. Phys. Vol.186 (1997), 731-750.

[5] A. Chamseddine, A. Connes, M. Marcolli, Gravity and the standard model with neutrino mixing, hep-th/0610241

[6] A. Connes, Noncommutative geometry, Academic Press (1994).

[7] A. Connes, Geometry from the spectral point of view, Lett. Math. Phys. 34 (1995), 203238

[8] A. Connes, Gravity coupled with matter and the foundation of noncommutative geometry, Comm. Math. Phys. (1995)

[9] A. Connes, H. Moscovici, The local index formula in noncommutative geometry, GAFA, Vol. 5 (1995), 174-243.

[10] A. Connes, On the spectral characterization of manifolds, arXiv Math.OA 0810.2088.

[11] J. Dixmier, Les $C^{*}$-algèbres et leurs representations Reprint of the second (1969) edition. Les Grands Classiques Gauthier-Villars. Éditions Jacques Gabay, Paris, 1996. 403 pp.

[12] C. Eckart, G. Young, A principal axis transformation for non-hermitian matrices, Bull. Amer. Math. Soc. 45, no. 2 (1939), 118-121.

[13] H. Figueroa, J.M. Gracia-Bondía, J. Varilly, Elements of Noncommutative Geometry, Birkhäuser, 2000.

[14] U. Haagerup, Orthogonal maximal abelian $*$-subalgebras of the $n \times n$ matrices and cyclic $n$-roots. Operator algebras and quantum field theory (Rome, 1996), 296-322, Int. Press, Cambridge, MA, 1997.

[15] H. B. Lawson, M-L. Michelsohn Spin geometry, Princeton Mathematical Series, 38. Princeton University Press, Princeton, NJ, 1989.

[16] J. Milnor, Eigenvalues of the Laplace operator on certain manifolds Proc. Natl. Acad. Sci. U S A. 51(4) (1964), 542.

[17] R. Nicoara, A finiteness result for commuting squares of matrix algebras. J. Operator Theory 55 (2006), no. 2, 295-310

[18] G. Pedersen, $C^{*}$-algebras and their Automorphism groups London Math. Society monographs 14. Academic Press (1979).

[19] S. Popa, Orthogonal pairs of $*$-subalgebras in finite von Neumann algebras. J. Operator Theory 9 (1983), no. 2, 253-268.

[20] A. Rennie, J.C. Varilly, Reconstruction of manifolds in noncommutative geometry, math.OA/0610418

[21] J. von Neumann, Zur Algebra der Funktionaloperatoren und Theorie der normalen Operatoren, Math. Ann. 102 (1929) 370-427.

[22] J. von Neumann, On rings of operators: reduction theory, Ann. Math. 50 (1949) 401-485. 
[23] T. Sunada, Riemannian coverings and isospectral manifolds. Ann. of Math. (2) 121, no. 1, (1985), 169-186.

[24] J. Sylvester, On the reduction of a bilinear quantic of the n'th order to the form of a sum of $n$ products by a double orthogonal substitution, Messenger of Mathematics, vol. 19 (1889), $42-46$.

[25] H. Taber On the linear transformations between two quadrics, Proceedings of the London Mathematical Society, vol. 24 (1892-93), 290-306.

A. Connes: Collège de France, 3, rue d'Ulm, Paris, F-75005 France, I.H.E.S. and VanDERBILT UNIVERSITY

E-mail address: alain@connes.org 